\title{
Computational generation of open-foam representative volume elements with morphological control using distance fields
}

\author{
N. G. Kilingar ${ }^{\mathrm{a}, \mathrm{b}}$, K. Ehab Moustafa Kamel ${ }^{\mathrm{b}}$, B. Sonon ${ }^{\mathrm{b}}$, T. J. Massart ${ }^{\mathrm{b}}$, L. Noels ${ }^{\mathrm{a}, *}$ \\ ${ }^{a}$ Computational \& Multiscale Mechanics of Materials (CM3), Department of Aerospace and \\ Mechanical Engineering, University of Liège, Quartier Polytech 1, Allée de la Découverte 9, B-4000 \\ Liège, Belgium \\ ${ }^{b}$ Building, Architecture \& Town Planning Department (BATir), Université Libre de Bruxelles (ULB), \\ CP 194/02, Avenue F.D. Roosevelt 50, 1050 Bruxelles, Belgium
}

\begin{abstract}
This paper presents an automated approach to build computationally Representative Volume Elements (RVE) of open-foam cellular materials, enabling the study of the effects of the microstructural features on their macroscopic behavior. The approach strongly relies on the use of distance and level set functions. The methodology is based on the extraction of random tessellations from inclusion packings following predetermined statistical packing distribution criteria. With the help of simple recombination operations on the distance fields, the tessellations are made to degenerate in Laguerre tessellations. Predetermined morphological characteristics like strut cross-section variation based on commercially available materials are applied on the RVE to ensure the extraction of closely matching models using simple surface extraction tools, and a detailed morphology quantification of the resulting RVEs is provided by comparing them with experimental observations. The extracted RVE surface is then treated with smoothening criteria before obtaining a 3D tetrahedralized model. This model can then be exported for multi-scale simulations to assess the effects of microstructural features by an upscaling methodology. The approach is illustrated by the simulation of a compression test on an RVE incorporating plasticity with geometrically non-linear behavior.
\end{abstract}

Keywords: Open-cell foam, Representational Volume Elements, Distance Fields

\section{Introduction}

Cellular microstructure in materials is one of the structural mechanisms permitting simultaneous optimization of stiffness, strength and overall weight in a given application. Such microstructures are naturally met in materials like cork, wood, bone and other cellular materials. These cellular properties enable materials in nature to exhibit

\footnotetext{
*Corresponding author, Phone: +324366 48 26, Fax: +3243669505

Email addresses: ngkilingar@uliege.be (N. G. Kilingar), thmassar@ulb.ac.be (T. J. Massart), 1.noels@ulg.ac.be (L. Noels)

$U R L:$ https://batir.ulb.ac.be/index.php (T. J. Massart), http://www.ltas-cm3.ulg.ac.be/index.html (L. Noels)

Preprint submitted to European Journal of Mechanics A/Solids

August 29, 2019
} 
superior performance for insulation, permeability or to support large loads [1, 2]. This has triggered the development of a class of light-weight cellular metallic open-foam materials with applications ranging from light-weight energy absorbers for impact loading applications and blast loading in the automotive [3, aerospace [4] and other industrial sectors [5, 6] and more recently in the field of thermal energy storage [7, 8, heat sinks [9, 10] and hydrogen production [11] among others.

Metallic open-cell foams can be manufactured indirectly from molds left after thermal treatment of reticulated polymer foams using investment casting, or by electro-deposition onto a polymeric foam with open cells, which is later removed resulting in hollow struts 3. The resulting foams in general follow the morphological characteristics of the polymeric foam from which they are derived. In general, they have a complex microstructure consisting of an interconnected network of ligaments forming along the edges of randomly packed cells that evolve during the foaming process of the polymeric foam [12]. A near equivalent to this type of material is a reticulated version of the equilibrated liquid foam, like that of soap froth or dry soap foam, where the cells that are near-polyhedral volumes originate from bubbles that are separated by tensioned liquid films. Under conditions of mechanical equilibrium where the surface free energy is minimized, the local foam geometry obeys Plateau's laws [13, 14] with constant mean curvature of each face, with three faces meeting at equal dihedral angles of $120^{\circ}$ at each cell edge, and with four edges joining at equal tetrahedral angles of $\cos ^{-1}(-1 / 3)=109.47^{\circ}$ at each cell vertex. Since the polymer open-cell foam is obtained by foaming a liquid polymer, the polymer-based mold presents morphologies strongly similar to liquid foams.

The link between the microstructure of foams and their physical behavior, like mechanical, acoustic, or thermal response, has been the focus of many studies. A number of experimental studies addressed this aspect in the literature [15, 16, 17, 18, 19. Along with these, computational models at the microstructural scale, or homogenization-based and multi-scale models, help in building an in-depth knowledge of the relationship between local morphological features and the global behavior of these materials. A model structure, with repeated calculations with varying model parameters, allows investigating the changes in the material properties associated with changes in microstructure. In his regard, 3D characterization and modeling of real structural foams using computer tomography $(\mathrm{CT})$ images can provide significant insight into the sample structure. These images can then be discretized and studied using FEM simulations [20, 21, 22. Yet, this remains a daunting task since characterizing all the samples requires both time and processing capacity, thus restricting the analysis to smaller samples requiring user-assisted analysis of the images [23].

Idealization of the microstructure geometry provides another important class of models that helps exploring the elementary mechanisms strongly related to the morphology of the material. Highly deterministic models based on observations from liquid foams have been studied. The Kelvin model 24] consists of a regular packing of slightly curves tetrakaidecahedra cells, for which the aim was to partition a three-dimensional space with minimum partitional area. This was next improved by the Weaire-Phelan foam [25]. A simplistic model made of rectangular prisms was proposed in [2]. These models contain a high degree of periodicity, and are thereby not able to capture the influence of the microstructural features on the mechanical properties due to the high degree of randomness in the real microstructures. An analysis of a tetrakaidecahedron unit cellbased computational micro-mechanics model with added imperfections was studied in 
[26] which predicted values that were in good agreement with the experimental data during the elastic response and the plateau stage. But even then, this geometry is not a reliable comparison.

The effect of variations on the overall mechanical properties caused by disorder in cellular solids induced by 'imperfections' with respect to an ideal structure required the introduction of complex three-dimensional random models to improve predictions [27. Random models of open foams inspired from a Voronoï tessellation of space based on the distributions of the nuclei 28 , or based on its weighted form called Laguerre tessellation [29, 30, were found to yield more realistic and versatile representations. Laguerre tessellations generated by random packings of spheres fitted to a given foam require an understanding of the dependence of geometric characteristics of the cells of the model on parameters such as the volume fraction (loosely packed or densely packed), or the radius distribution of the sphere packing (normal distribution, log-normal distribution, gamma distribution,...). A lot of studies have been carried out to fit models according to the material characterizations 31, 32. The statistical nature of these microstructures implies that even though two structures obtained under same modeling conditions differ locally in their geometry, for relatively large samples, their statistical descriptors, such as the average pore size and pore size variations are the same. This allows establishing statistical relationships among some relevant features of these structures like variability of pore volume.

A wide range of sphere packing algorithms, like force biased algorithm or Jodrey-Tory gravitational algorithm, both classified under collective rearrangement, sedimentation algorithms, or sequential addition methods, exist to simulate spheres packings 33. Studies have also targeted the understanding of the effect of surface minimization principles to obtain equilibrated shapes in the foam model with Plateau borders undergoing topological evolutions on Laguerre tessellations using the Surface Evolver software [14, 34, 35, 36]. These generation methods are able to properly represent the foam morphological parameters like the cell size distribution, the face-by-cell count, and the edge-by-face count. However, the tessellations cannot represent local morphological features related to the strut, like the strut cross-sections shape, variation and curvature, the coating over the strut, or the presence of hollow struts. The existence of partially reticulated (intermediate state between completely open and closed foams) cannot be accounted for using simple tessellations, requiring further constructions [12]. Depending on the process used and on requirements, open foam materials are produced with varying strut cross-section, ranging from concave plateau borders to circular shapes that result in the increase of the relative density and variation of cross-section along their length with more material at the nodes 12 .

The morphological values of the foam seem to depend on the source and the manufacturing process involved to obtain these samples and hence, a lot of variations can be found in existing publications in their quantification. It is thus of interest to implement a generation tool that can be versatile in its use and take into account these variations. For example, the porosity of the foam decreases with an increase in the pores per inch (ppi) value of the foam under study [37. This could be explained by the increase in the thickness of the struts forming the foam due to the completion of the solidification process before equilibrium can be reached. Since these geometrical features can drastically influence the mechanical behavior of the foam [37, 38, it thus becomes necessary to capture the variations in the strut morphology to generate numerical models under the 
form of Representative Volume Elements (RVEs) that can mimic real foam samples to a high degree of accuracy and without utilizing much computational power for the RVE generation process itself. In [39, open-foam RVEs were generated from Surface Evolver. However, these models need to be built strut by strut and a special treatment is required to account for the accumulation of material at the strut junctions due to intersection of these struts.

A efficient RVE generation tool was presented by Sonon et al. [40] based on the concept of level set functions and distance fields. The tool utilizes a classical Random Sequential Addition approach for inclusions packing, combined with a level set function control on the process resulting in a linear dependence of the addition cost in terms of the number of inclusions instead of exponential dependence. By introducing simple functions based on the distance fields to neighboring inclusions, the morphologies of cellular open foam materials based on tessellations can be built, taking into consideration the strut characteristics based on introducing controlled variability on parameters governing the resulting microstructural morphologies [41. The tool makes use of discrete grids for the evolution of distance functions to assign distance values to the sphere to all the points in the domain based on their proximity to the nearest neighbors. However, the discrete nature of these function evaluations brings its own set of problems, i.e., the inability to resolve sharp edges of the foam structures using single level sets or distance functions.

In this paper, we look at producing morphologies of open foam materials starting from the RVE generation tool developed in [40. We also recall the tools to extract inclusion surfaces using a "Plateau" level set function for all inclusions in the RVE focusing on the proper reproduction of the sharp edges of the struts involved. We also discuss the extraction of additional critical features of the foam like open/close intermediate faces, strut concavity and cross-section variation of the extracted foam, while devising a methodology to extract the strut cross-section and the thickness variation along the length of the strut. Once the sharp edged struts with their respective morphologies are extracted, a finite element discretization of the obtained implicit geometry is performed based on an extension of the works of Persson [42, resulting in conforming tetrahedral meshes of open foam structures. A selective assessment of some of the morphological indicators of the foam, like the face-by-cell count, the edge-by-face count and the strut length distribution, is performed next based on the studies conducted in literature [31, 36, 37, 43, showing that these properties are satisfactorily reproduced compared to the ones found in existing models generated by random Laguerre tessellations. A particular focus is set on properties related to open-cell aluminum foams with varying pore sizes. This example is used to demonstrate the flexibility of the method to account for detailed micro-structural features such as strut shape variations, with a computational efficiency enabling the generation of a large number of volume elements of arbitrary size for finite element simulations.

The paper is outlined as follows: In Section 2, basics and notations of the DNRSA tool described in 40] and 41] will be recalled. Section 3 details the various foam morphologies including strut cross-section variation and the various functions necessary to extract them using distance functions. Section 4 presents the method to extract the sharp edges from the distance functions using multiple level sets. In Section 5, the steps to obtain a finite element mesh from the implicit geometry of the open-foam RVE will be presented. Section 6 describes the quantification of the obtained morphologies, the tessellations and the strut morphologies. Section 7 presents the results of numerical 


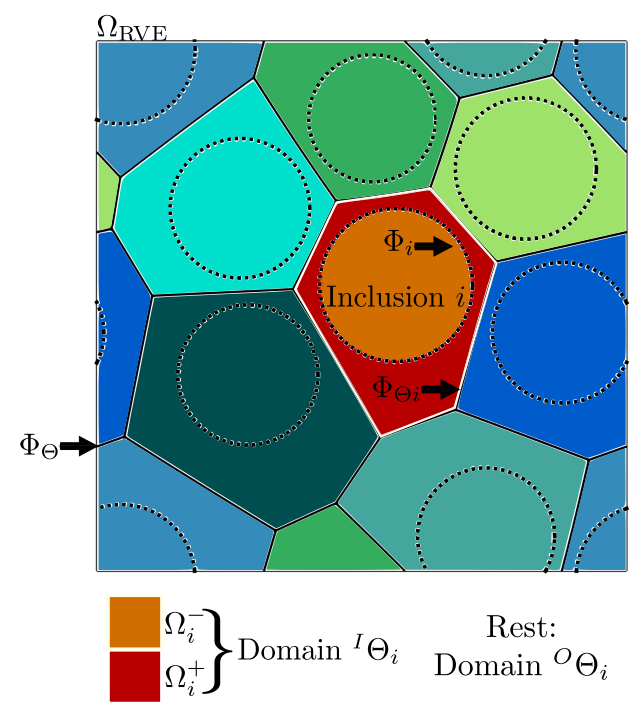

Figure 1: Domain definitions; Dashed lines denote inclusion boundaries while bold lines denote $\Phi_{\Theta}$.

simulations on the RVEs generated showing the ability of the framework to reproduce real foam behaviors.

\section{Open foam morphology generation}

We start from the previous work done in 40 that explains the building of an arbitrary shaped tessellation with the help of neighboring distance fields of an inclusion packing, and from the reference [41] that explains the basis of the approach to generate RVEs for open and closed foam morphologies. For the sake of clarity and completeness we recall the methodology that will help in understanding the approach.

\section{1. $D N-R S A$}

In classical Random Sequential Addition (RSA), the rate of rejection of the potential position of a new inclusion to be added increases when the volume fraction increases and/or when the need for a neighboring distance control leads to very restrictive tests. To overcome this drawback, Sonon et al. have proposed in [40] an RVE generation tool to introduce arbitrarily shaped inclusions in the RVE domain with the help of distance fields indicators. The tool introduces a neighboring distance control at each point of a discrete grid using the distance field to the $k$-th neighboring inclusion, $D N_{k}(\mathbf{x})$, denoted as $D N$ finder or Distance-Neighbor finder. Since the method rests on an extension of the classical Random Sequential Addition approach this tool can be called Neighboring distance controlled RSA, or DN-RSA approach. We will briefly recall the nomenclature that will be used in the following sections.

If we consider a domain $\Omega_{\mathrm{RVE}}$ (Figure 1), where we want to introduce an inclusion $i$, member of the set $\mathbf{I}$ of all inclusions, then $\Omega_{i}^{-}$represents the domain inside the boundary of the inclusion denoted by $\Phi_{i}$ and $\Omega_{i}^{+}$the domain outside $\Phi_{i} . D S_{i}(\mathbf{x})$ is defined as the 


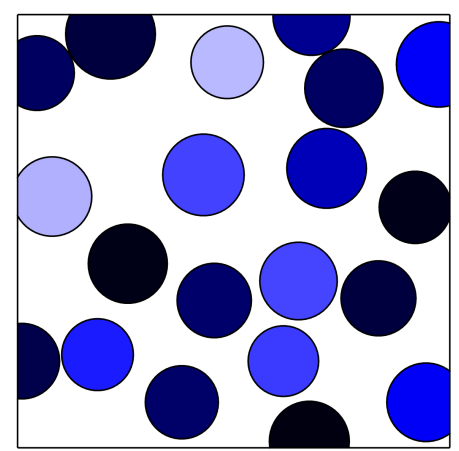

(a)

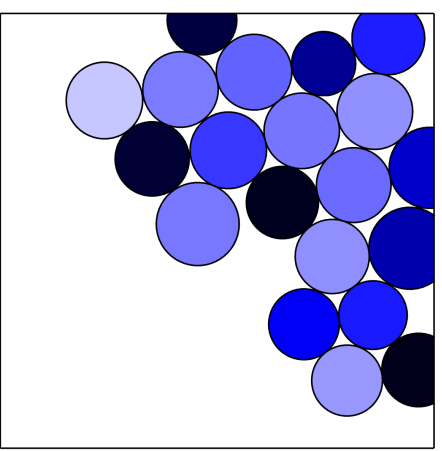

(b)

Figure 2: Effect of $n n l$ on the packing of an RVE with 20 inclusions; (a) Non-overlap control only, and (b) enforcing a minimal neighboring distance.

signed distance field of $i$, with negative value in $\Omega_{i}^{-}$and positive value in $\Omega_{i}^{+}$. At every position $\mathbf{x}, D N_{k}(\mathbf{x})$ gives the distance from $k$-th nearest $\Phi_{i}$ in the packing and thus, all inclusions in the packing can be represented by using $D N_{1}(\mathbf{x})$ as a level set. $N N_{k}(\mathbf{x})$ denotes the $k$-th neighbor identity map. As an integer discontinuous function, at each point in the domain, $\mathbf{x}$, it denotes the nearest inclusion from $\mathbf{x}$ in the set $\mathbf{I}$. $\mathbf{J}_{k}(\mathbf{x})$ defines an $\mathbf{x}$ dependent set constructed from $\mathbf{I}$ excluding all $N N_{m}, m \in[1: k-1]$. This quantity is computationally free and helps constructing $D N_{k}(\mathbf{x})$ as:

$$
D N_{k}(\mathbf{x})=\min _{j}\left(D S_{j}(\mathbf{x})\right), \text { with } j \text { in } \mathbf{J}_{k}(\mathbf{x})
$$

We further define ${ }^{I} \Theta_{i}$ as the "Inner" domain where inclusion $i$ is the first nearest inclusion. It is the set of points $\mathbf{x}$ closer to inclusion $i$ than to any other inclusion, i.e. where $N N_{1}(\mathbf{x})=i, \forall \mathbf{x} \in{ }^{I} \Theta_{i}$. Similarly, ${ }^{O} \Theta_{i}$ is the "Outer" domain where $N N_{1}(\mathbf{x}) \neq i$. $\Phi_{\Theta i}$ is the boundary of ${ }^{I} \Theta_{i}$ and $\Phi_{\Theta}$ is the union of all $\Phi_{\Theta i}$.

In RSA, the rejection rate of an inclusion is reduced with an a priori knowledge of sub domains where the position of the inclusion will respect all the criteria of a positioning test. Logical operations on the $D N_{k}$ functions can help in selecting a large range of discrete structured or unstructured positions in the RVE discretizing the description of the neighboring distance functions. For arbitrary shaped inclusions, the smallest enclosing circle/sphere (SEC / SES) of radius $r$ determines the non-overlap criteria on any inclusion in the RVE already mapped in $D N_{1}(\mathbf{x})$ when the condition

$$
D N_{1}\left(\mathbf{x}_{c}\right)>r
$$

is satisfied for its center $\mathbf{x}_{c}$. In the case of circular/spherical inclusions, $r$ is a random variable with $r \sim R, R$ being a radius distribution (like normal, log-normal or gamma distributions).

A control on the distances from the first, second (for $2 \mathrm{D}$ circles) and / or third (for $3 \mathrm{D}$ spheres) nearest neighbors (denoted by $n n l_{1}, n n l_{2}$ and $n n l_{3}$ in the sequel) can help produce closer packings, thus enabling higher packing densities (Figure 2). Taking 


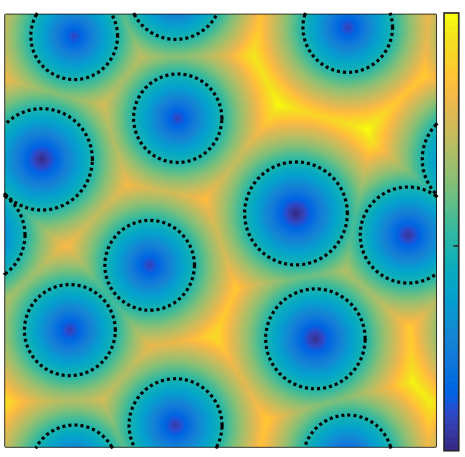

(a)

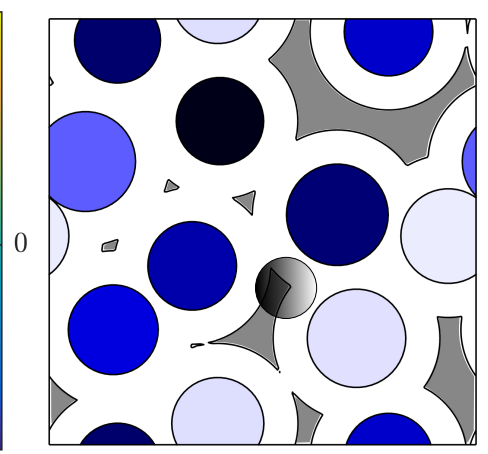

(b)

Figure 3: (a) $D N_{1}$ function plot. (b) Using Eqs. 20 or 3 the shaded domain can be extracted where the criteria for the next inclusion is satisfied.

inspiration from algorithms like the clustering approach [44] and the advancing front [45], where the inclusions are placed on the vertices of triangles (2D) or tetrahedra (3D) to position the new inclusion close to the existing two or three nearest neighbors, the condition

$$
D N_{k}\left(\mathbf{x}_{c}\right)<n n l_{k}+r
$$

is introduced, where

$$
k=\left\{\begin{array}{l}
\{1 ; 2\} \text { in } 2 \mathrm{D} \\
\{1 ; 2 ; 3\} \text { in } 3 \mathrm{D}
\end{array}\right.
$$

Higher $k$ values can also be considered which can then be used for various operations on the morphologies, such as the one described in Section 3.2

Thus, the DN-RSA can be summarized as follows:

1. Construct a discretized RVE domain made of a regular grid of points $\mathbf{x}_{n}$ and initialize $D N_{k}\left(\mathbf{x}_{n}\right)$ to $+\infty$.

2. Generate trial inclusion $i$ from prescribed size distribution function / predefined set.

3. Use condition (2) to avoid inter-penetration and condition (3) to meet neighbor distance criteria, retaining all satisfactory $\mathbf{x}_{c}$ (Figure 3). If this is empty, the RVE is full for the inclusion size considered, terminating the process.

4. Refresh $D N_{k}\left(\mathbf{x}_{n}\right)$ to allow the calculation of $D S_{i}\left(\mathbf{x}_{n}\right)$ of new inclusion $i$ using a sequential adaptive refreshing.

5. Follow step 2 until termination criteria is reached or the RVE is full (Figure 4).

To increase the computational efficiency of the $D N_{k}$ evaluations, a sequential adaptive scheme is introduced. In such a scheme the contribution of each $D S_{i}$ is introduced simultaneously in functions $D N_{k}$ as inclusions are introduced sequentially along time represented by an index $m$, using:

$$
\begin{gathered}
D N_{1}(\mathbf{x})^{m}=\min \left(D N_{1}(\mathbf{x})^{m-1}, D S_{i}(\mathbf{x})^{m}\right), \\
D N_{k}(\mathbf{x})^{m}=\max \left(D N_{k-1}(\mathbf{x})^{m-1}, \min \left(D N_{k}(\mathbf{x})^{m-1}, D S_{i}(\mathbf{x})^{m}\right)\right) . \\
7
\end{gathered}
$$




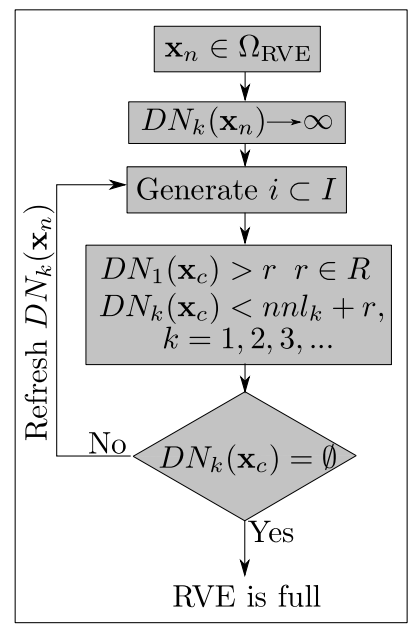

Figure 4: DN-RSA process.

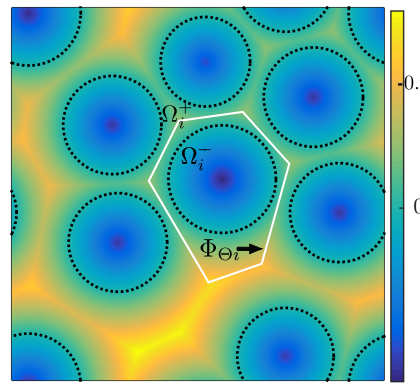

(a)

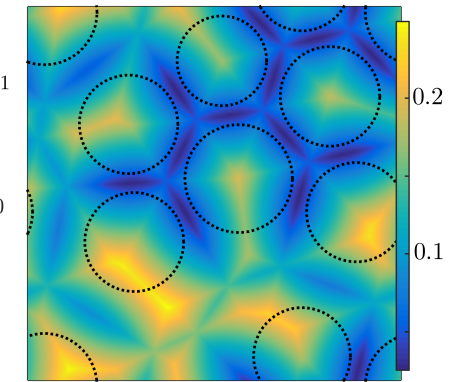

(b)

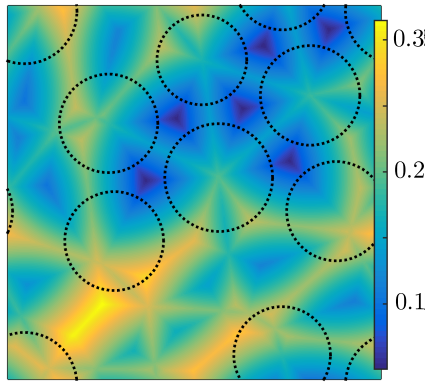

(c)

Figure 5: Functions (a) $D N_{1}$, (b) $D N_{2}$, and (c) $D N_{3} . \Phi_{\Theta i}$ is marked for comparison.

The differences in the first three $D N_{k}$ functions have been illustrated in Figure 5 . In all illustrations provided in this paper, RVEs are considered with unit lengths in all directions. The scale reported with the $D N_{k}$ functions in Figure (5) should therefore be understood in relative terms with respect to the RVE dimensions.

Application of periodicity in the RVE boundaries may be advantageous in obtaining statistically homogeneous RVEs without border effects that introduce morphological difference between the bulk of the RVE and its boundary neighborhood. Also, homogenization techniques were shown to give better result while using periodic boundary conditions in the physical model [46] (Figure 6). Nevertheless, free boundary conditions in RVE geometries enable generating models free of constraints induced by the periodicity of the RVE. In this case, to avoid the spatial sampling bias introduced due to the interaction of the inclusions at the boundary with the domain $\Omega_{\mathrm{RVE}}$, minus sampling operation 47] can be considered where one samples only those objects that lie within a sampling frame (Figure 7). A maximum permissible unit length, which is smaller than 


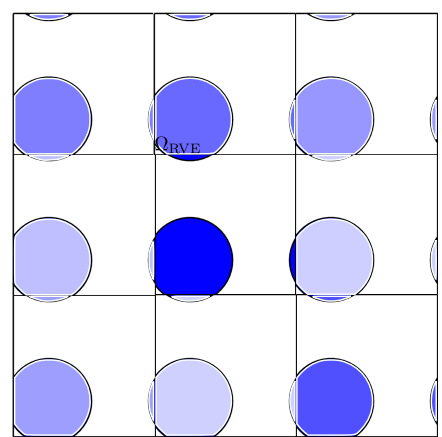

(a)

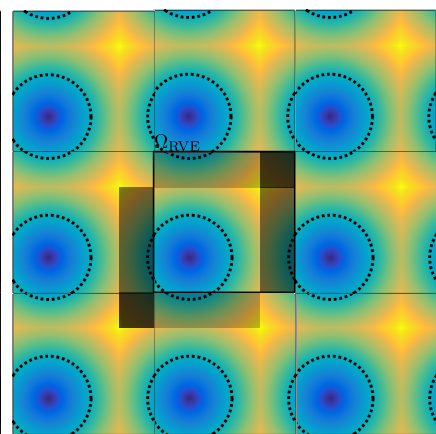

(b)

Figure 6: (a) Reproduction of an inclusion in its eight periodic neighbors; (b) $D N_{1}$ reproduction in the periodic setting. Periodicity is treated by extending the influence domain out of the RVE and by bringing them back in the RVE to its periodic location by translation.

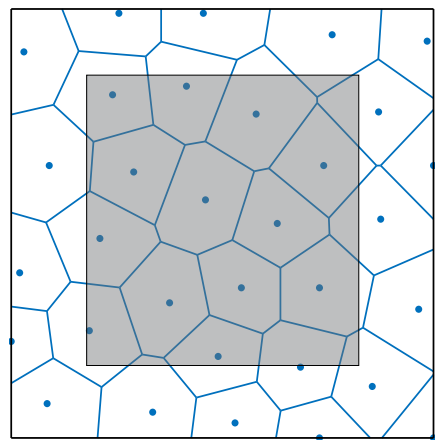

(a)

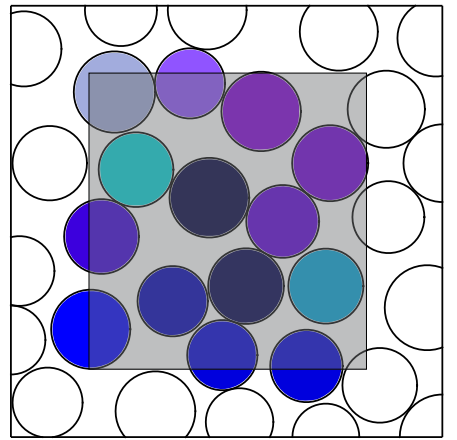

(b)

Figure 7: Minus sampling of free boundary RVE; (a) Only inclusions whose center lies within the domain definition are considered for treatment. (b) The new boundary of the domain and the objects that are taken into consideration.

the RVE dimensions, can be used such that the centers of all the objects whose $\Phi_{\Theta i}$ are completely inside the domain lie inside this limit length. This quantity can be defined as a certain fraction of the dimensions of the RVE depending on the average size of the inclusions. More details on considerations related to the evaluation of periodic $D N_{k}$ functions can be found in [48. It can be noted that the application of periodic boundary conditions on system made of nonconformal or free boundary geometry can be solved by using specialized methods for this purpose 49. The methodology to extract open-foam morphologies from the inclusion packings discussed in [4] is recalled next.

\section{2. "Voronoi" and "Plateau" level set functions}

Classical tessellation patterns usually consist of tiling of a plane using multiple geometric shape and the techniques used to generate these patterns gather cell points in each tessellation that are closer to a particular seed point. To this end, they require the use of distance measures. Every point in the domain will have one nearest seed, and con- 


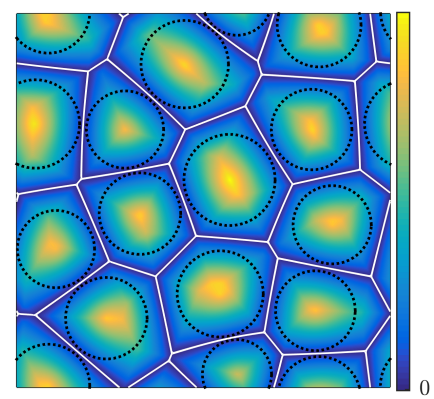

(a)

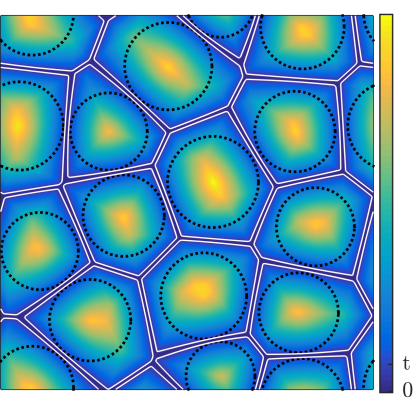

(b)

Figure 8: Voronoï level set functions for disk packings; (a) From Eq. 66, and (b) following Eq. 77 with $t=0.01$.

sequently each seed has one sub-domain generated. This ensures that the sub-domains are exactly adjacent to each other. The Voronoï tessellation uses points as seeds and the Euclidean distance as distance measure with additively weighted Voronoï tessellation using circular/spherical seeds and Laguerre tessellation using sphere/circle power distance on a spherical/circular packing. The Laguerre tessellations are usually preferred as multi-sized cell tessellations (polydispersity) can be easily produced while keeping the cell faces plane and the edges of the cell faces straight (lack of edge curvature is an observed phenomenon in real foams [50]).

With the help of notations in Figure 1, from a given inclusion packing generated by DN-RSA, it is possible to define a tessellation made by the assembly of all ${ }^{I} \Theta_{i}$ domains which degenerates in a Laguerre tessellation for multi-sized sphere packings. This tessellation can be constructed implicitly with the first and the second neighbor distance fields of the initial inclusion packing by a "Voronoi" level set function defined as:

$$
O_{V}(\mathbf{x})=D N_{2}(\mathbf{x})-D N_{1}(\mathbf{x})
$$

The value of this function is exactly zero at loci equidistant from two nearest inclusions, i.e. on the faces of the tessellations, and is positive everywhere else, as can be seen in Figure 8 a for a $2 \mathrm{D}$ example. A quasi constant thickness, $t$, can be used in combination with $O_{V}$ to extract a closed cell geometry through level sets:

$$
O_{V}(\mathbf{x})-t=0
$$

Figure $8 \mathrm{~b}$ also illustrates this relation in 2D. Figure $9 \mathrm{a}$ illustrates a packing generated in 3D, with the corresponding RVE generated by the use of Eq. (7) in Figure 9 s.

Open foam microstructures can be defined by the interlinking of 3D struts, usually with a triangular cross-section. An ad-hoc level set function can be defined in order to extract such struts from the edges of the tessellation. Using the first three neighbor distance functions, and taking inspiration from the knowledge that Plateau borders in liquid foams form at the intersection of three films, a relevant function denoted as the 


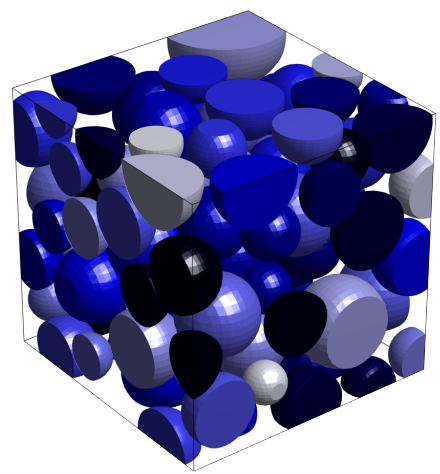

(a)

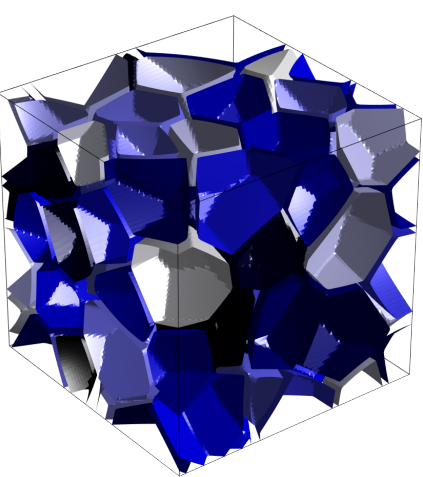

(b)

Figure 9: (a) A multi-sized sphere packing, and (b) a closed cell RVE generated from the packing with $t=0.025$.

"Plateau" level set function can be defined as:

$$
O_{P}(\mathbf{x})=\frac{\left(D N_{3}(\mathbf{x})+D N_{2}(\mathbf{x})\right)}{2}-D N_{1}(\mathbf{x}) .
$$

This function vanishes at the locus where the distance from the three nearest inclusions is the same, and is positive everywhere else.

The level set of this function consists of triangles with vertex lying on the tessellation cell boundaries. A natural way of extracting a Plateau border like geometry is therefore given by

$$
O_{P}(\mathbf{x})-t=0
$$

where the parameter $t$ controls the thickness of the extracted boundary (Figure 10a). This thickness is related to the parameter in Eq. (7) such that the plateau borders produced can be completely contained in the corresponding closed cell walls (Figure $10 \mathrm{~b}$. tions.

Figure 10c illustrates the example of an RVE in 3D extracted using the above rela-

\section{Detailed foam morphology features}

Realistic features that are seen according to CT scans in real foams can be introduced in RVEs with the help of functions built on the basis of the distance fields. The extraction of some of these characteristically important features in this section are based on the suggestions provided in [48, and an original methodology to extract complexities in strut geometry like the variation of strut cross-section is presented here.

\subsection{Open / close faces}

Observations from micro-CT have shown the existence of several sites in a metallic open cell foam where existing holes in the original polymeric template have been presumably filled in during the molding process [12]. Such sites of local material concentration 


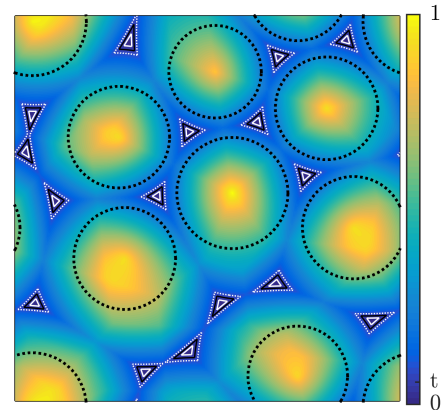

(a)

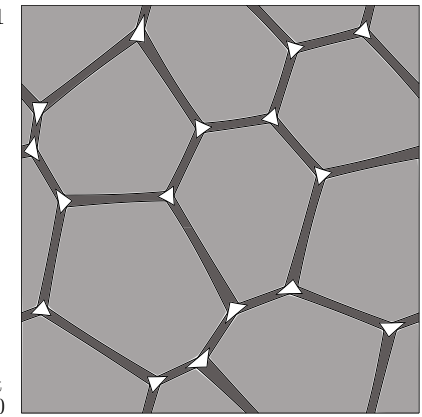

(b)

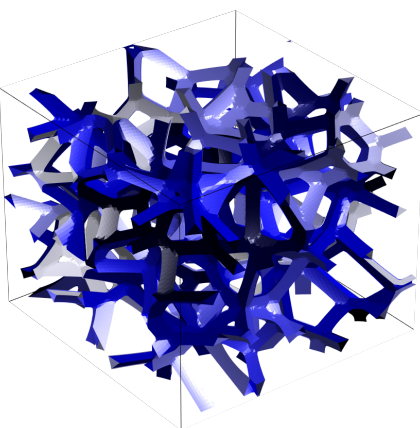

(c)

Figure 10: Plateau level set function; (a) with incremental $t$ value, of 0.01 denoted by white line, 0.02 by black line, and 0.03 by dotted white line displaying the triangular nature of the isolines extracted by the function, and (b) illustration of the relation between the $t$ level sets extracted from Eqs. 7) and (9); (c) Open foam extraction using Plateau level set function Eqs. (7) and (9) with $t=0.15$.

result in local stiffening and also contribute to the overall weight of the foam. With the help of a value $0<a<1$ defining the ratio of open/closed faces, such situations can be naturally achieved using a linear combination of $O_{V}$ and $O_{P}$ as described in [4]:

$$
a O_{V}(\mathbf{x})+(1-a) O_{P}(\mathbf{x})-t=0 .
$$

Figure 11 illustrates the use of this relation for two values of $a$.

\subsection{Strut cross-sectional area distribution}

Irrespective of the pore sizes, struts cross-sectional areas vary along their length. Material is concentrated in the nodes where the struts intersect while a lower material concentration is found at the mid span [37, 38]. In [12, ligament slicing operations have been used to evaluate the ligament cross-sectional area $A(\xi)$ normalized by the value at mid-span $A_{0}$ as a function of axial position $\xi=x / l, l$ being the ligament length:

$$
A(\xi)=A_{0}\left(c_{1} \xi^{4}+c_{2} \xi^{2}+1\right)
$$

with $c_{1}$ and $c_{2}$ fitting constants. The $\xi$ value varies from -0.5 to 0.5 . Varying the values of $c_{1}$ and $c_{2}$ enables controlling the density which helps achieving various ppi models in RVEs. The studies also suggest that the longer ligaments tend to have smaller $A_{0}$ than the shorter ones [12. Fitting of the data obtained by comparing $A_{0}$ against ligament length gives a function of the form:

$$
A_{0}(\eta)=\bar{A}_{0}\left(d_{1}+d_{2} \eta^{-\beta}\right) \text {, with } \eta=l / \bar{l},
$$

where $\bar{A}_{0}$ and $\bar{l}$ denote normalized mean values of all the measurements. For Al-6101-T6 Duocel open-cell foams made by ERG, the measurements were fitted with the values $c_{1}=36, c_{2}=1, d_{1}=0.6633, d_{2}=0.2648$ and $\beta=2.5963$.

These parameters can be easily represented implicitly using the $D N_{k}$ functions. The operator

$$
O_{S 1}(\mathbf{x})=D N_{4}(\mathbf{x})-D N_{3}(\mathbf{x})
$$




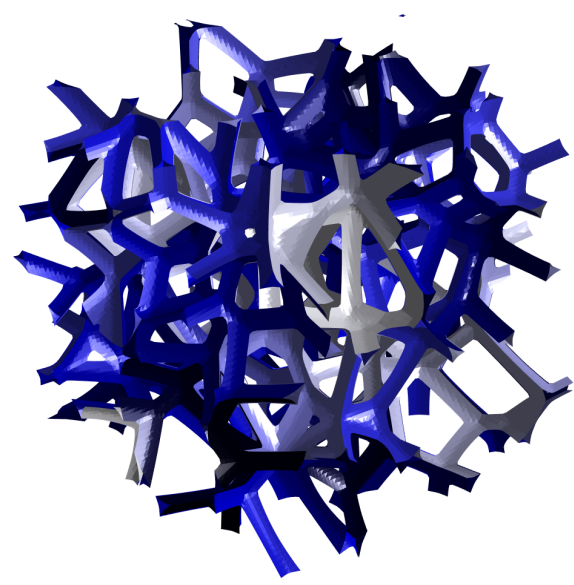

(a)

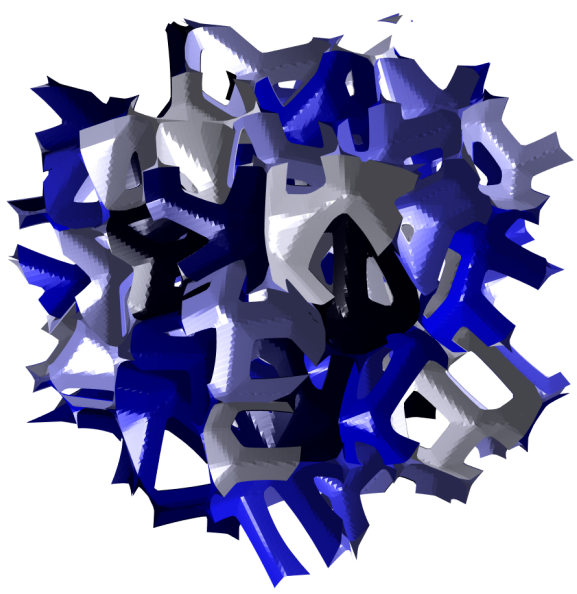

(b)

Figure 11: Open/Close intermediate situation using (a) $a=0.25$ and (b) $a=0.5$.

varies according to the distance from the nearest node and thus, along the struts, its value increases from 0 at the nodes to half the strut length at its mid-section. If we consider the domain

$$
\Omega_{i j k}=\left(N N_{1}(\mathbf{x})=i\right) \&\left(N N_{2}(\mathbf{x})=j\right) \&\left(N N_{3}(\mathbf{x})=k\right),
$$

this domain represents a tetrahedral part of the tessellation cell with the vertices lying at the center of the cell generated by inclusion $i$, the center of the face shared with the cell generated by the adjacent inclusion $j$, and the two ends of the strut with plateau border due to the cell generated by inclusion $k$. The maximum value of $O_{S 1}$ in $\Omega_{i j k}$ is equal to half the length of the corresponding strut. We can therefore replace $\eta$ in Eq. (12) by

$$
\eta=\frac{\max O_{S 1}\left(\Omega_{i j k}\right)}{\operatorname{mean}\left(\max O_{S 1}\left(\Omega_{\mathrm{RVE}}\right)\right)},
$$

and one can reformulate $\xi$ in Eq. (11) as

$$
\xi^{\prime}=\frac{O_{S 1}\left(\Omega_{i j k}\right)}{\max \left(O_{S 1}\left(\Omega_{i j k}\right)\right)},
$$

with $\xi^{\prime}$ value varying from 0 at the nodes to 1 at the mid-span of the strut. Since this function is defined as a three dimensional one, a simple visualization is difficult to extract, but a limited visualization on the resulting isosurface of an inclusion is given in Figure 12 .

Using the reformulated definitions and applying the new limits, the strut cross-section variation given by Eq. (11) can be reformulated as

$$
A\left(\xi^{\prime}\right)=A_{0}\left(e_{1} \xi^{\prime 4}+e_{2} \xi^{\prime 3}+e_{3} \xi^{\prime 2}+e_{4} \xi^{\prime}+e_{5}\right),
$$




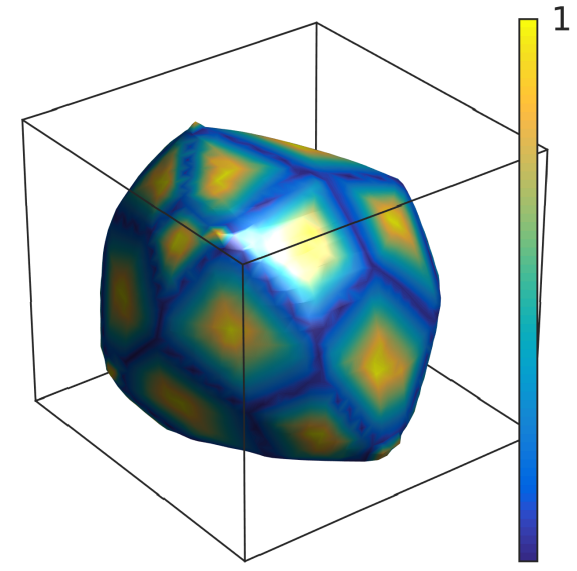

(a)

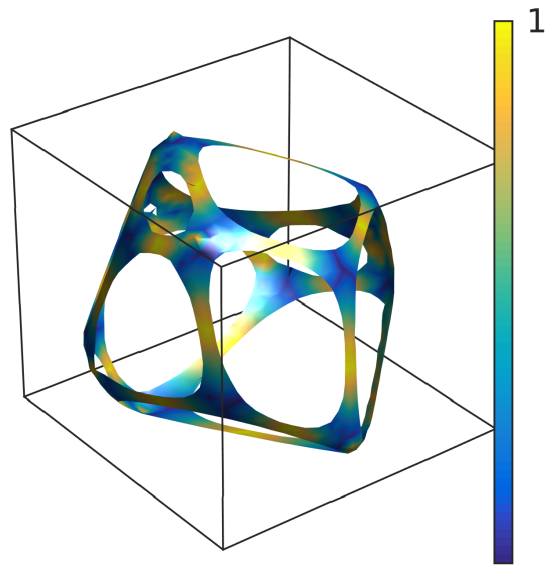

(b)

Figure 12: The variation of $\xi^{\prime}$ on the isosurface extracted using Eq. 18. The variation of $\xi^{\prime}$ from 0 to 1 along the strut surface can be observed. A sliced surface of the same inclusion is given for comparison.

with $e_{m}, m=1,2, \ldots, 5$, as the reformulated coefficients of the function with changed limits. We introduce a global operator

$$
O_{S}(\mathbf{x})=\sqrt{\frac{A\left(\xi^{\prime}\right)}{A_{0}}}
$$

and combine it with Eq. (9) to control the variation of the strut cross-section in the generated morphology as:

$$
O_{P}(\mathbf{x})-t . O_{S}(\mathbf{x})=0 \text {. }
$$

Figure 13 shows the effect of introducing the parameters on the foam morphology and the corresponding variations in the weight density of the RVE. This enables controlling the ppi ratio with the commercially available foams, while maintaining the unit dimensions of the generated RVE necessary to perform a numerical analysis.

\subsection{Strut concavity}

Polyurethane (PU) ligaments usually have a 3-cusp hypocycloid cross-section varying along the length of the strut, while most of the metallic foams have a triangular cross-section that tends towards circular with increasing ppi value. To obtain such morphological variations, we can subtract a non-constant $C^{2}$ continuous function that is free from curvature jumps and unwanted sharp edges from the function defined in Eq. (18). Such a function can be defined based on the maintained distance fields according to:

$$
O_{K}(\mathbf{x})=\min \left(0, \frac{\left(\left(\frac{D N_{3}(\mathbf{x})-D N_{2}(\mathbf{x})}{2}\right)^{2}-\left(t . O_{S}(\mathbf{x})\right)^{2}\right)}{2 t \cdot O_{S}(\mathbf{x})}\right),
$$




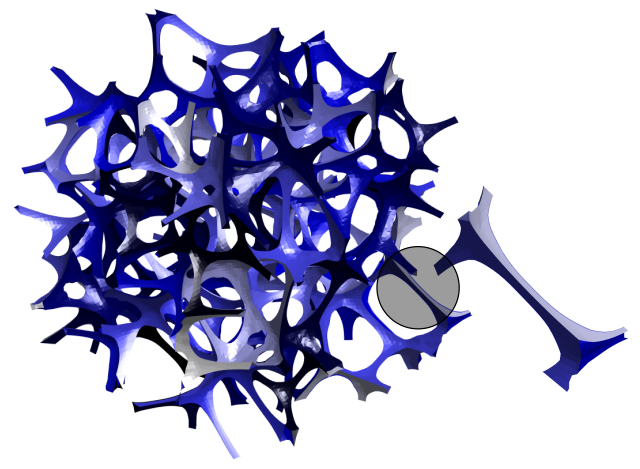

(a)

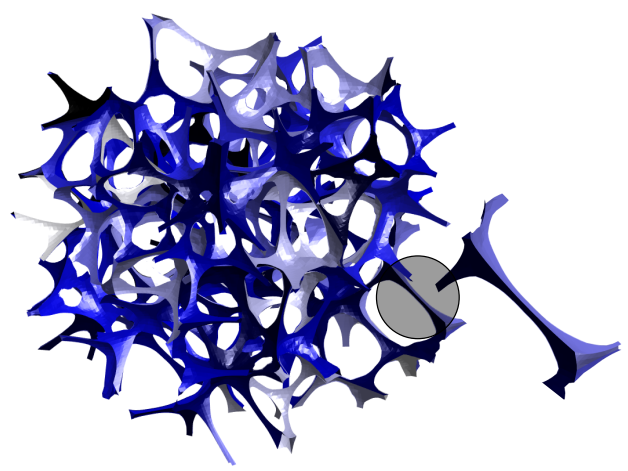

(b)

Figure 13: Effect of Eq. 111 on the strut cross-section with (a) $c_{1}=20$ and $c_{2}=1$, and (b) $c_{1}=50$ and $c_{2}=1$.

which can now be combined with Eq. (18) to control the plateau border concavity by using:

$$
O_{P}(\mathbf{x})-t . O_{S}(\mathbf{x})-k_{c} \cdot O_{K}(\mathbf{x})=0
$$

with $0<k_{c}<1$ as the concavity controlling parameter. The effect of concavity as well as of strut cross-section variation is illustrated in Figure 14 . Operator 20 is acceptable for simple cross-sectional geometries like triangular, concave triangular or circular shapes. Slight variations with $k_{c}<0$ can also be studied to analyze RVEs with struts having convex triangular cross-sections, as it will be illustrated in Section 6 .

\subsection{Anisotropy}

The metallic foams manufactured by investment casting over a PU base display anisotropic characteristics in the rise direction [12. This anisotropy can be implemented in the RVE using DN-RSA in the initial packing generation by the use of anisotropic packings instead of spherical packing. An anisotropy parameter can also be introduced to the distance functions, which is simply the percentage of the original length, once the packing is generated, in a particular direction so that the RVE generated using the function $O_{P}$ leads to the extraction of an anisotropic RVE, as it will be illustrated in Section 6. Anisotropy in foam can also exist in the form of partially reticulated, or intermediary states, between open and closed walls in some of the pores 39, the implementation of which can be found in [40].

\section{Sharp edge extraction by means of local level set function}

The steep discontinuity of $D N_{k}$ derivatives on $\Phi_{\Theta}$ cannot be handled by the use of level set functions evaluated on discrete grids of points, and thus the sharp edges of the Plateau borders geometry cannot be extracted with simple contouring algorithms (Figure 15a). This problem is illustrated in Figure 15b where jagged edges are obtained when a classical contouring is applied. This prevents the discretization by means of a proper finite element mesh. To overcome this issue, the global geometry can be split 


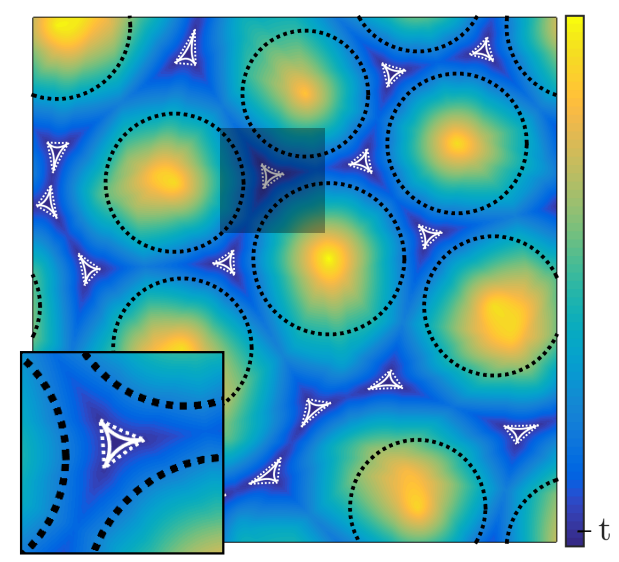

(a)

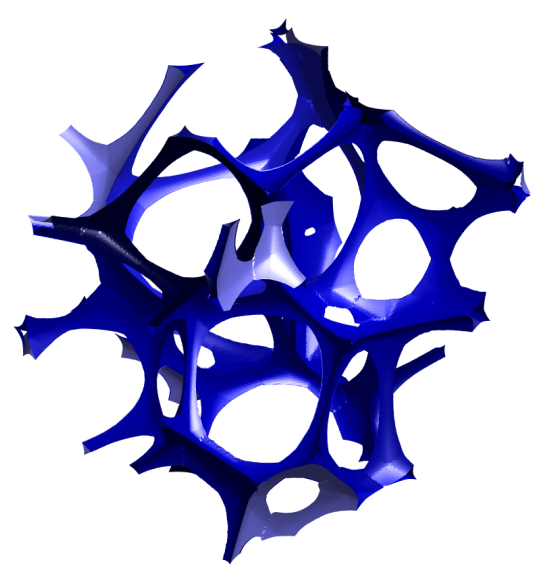

(b)

Figure 14: Plateau border concavity control. (a) White lines denote concavity with $k_{c}=1$ while dashed lines denote simple plateau border, and (b) $3 \mathrm{D}$ detail with $k_{c}=0.5, c_{1}=20$ and $c_{2}=1, d_{1}=0.6633$, $d_{2}=0.2648$ and $\beta=2.5963$, the data being obtained from statistical analysis of the entire foam sample [12].

into an assembly of smooth surfaces extracted from separate "Local" level set functions as proposed in [41. By constructing an individual local level set for each inclusion, the problem arising due to sharp edges can be avoided (Figure 16). However, in order to obtain the holes on the resulting surfaces that exist in the open foam, additional slicing operations need to be carried out. Two methods can be suggested for such an operation:

1. Construction of a second "outer" level set function for each inclusion which is used to slice the surface obtained by the first "inner" level set function. By interpolating the values of the second function on the vertices of the surface obtained from the first function, the slicing operation can be carried out using a "marching algorithm" 41. When using this method, once the surface coming from inclusion $i$ is extracted, it is not necessary to store both "inner" and "outer" functions in memory. For large RVEs though, especially in 3D, this creates the issue of mismatch of vertices of a common sharp edge between two due to the discrete evaluation of the extracted functions. In such cases, for classical contouring implementations, there is no guarantee that the two smooth surfaces belonging to adjacent inclusions meeting at the sharp edge will have the same discretization at their common boundary.

2. The surface of an inclusion can be sliced by comparing it with the surface of the neighboring inclusion using Boolean intersection of surfaces. This operation, while using the same concept as in option 1, results in the generation of surfaces with holes that form the sharp edges of the struts, perfectly aligned with the corresponding sharp edge from the surface of the neighboring inclusion. This approach is slightly more costly in comparison with the first method because, for each inclusion, all the neighboring "local" functions have to be called iteratively. However, it is advantageous as the surfaces extracted for two neighboring inclusions would have common edge vertices, facilitating the post processing in the form of mesh refinement of 


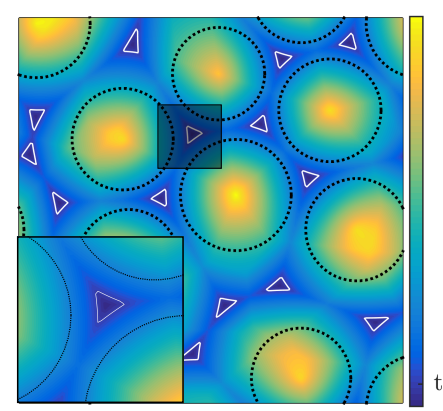

(a)

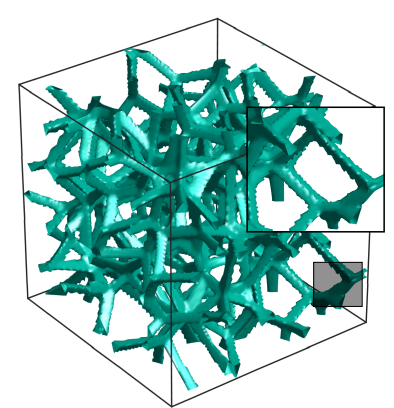

(b)

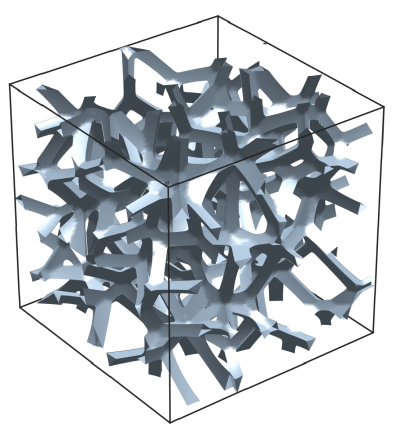

(c)

Figure 15: Sharp edges extraction; Result of a single level set function extraction resulting in a blunted or jagged geometry in (a) disk packing, and (b) sphere packing; (c) RVE after multiple level set extraction. Strut morphology variations are not implemented in this illustration.

these edges. This second approach will be used in the following sections.

To achieve this enhanced slicing, a "local" level set function for each inclusion is built by modifying the "global" $D N_{k}$ functions, and is further denoted as ${ }^{I} D N_{k i}$. Without changing the definition of $O_{P}$, a "local" ${ }^{I} O P_{i}$ is built with ${ }^{I} D N_{k i}$ using Eq. 88). This "local" level set is used to extract the surface coming from the considered inclusion, while similarly built "local" level sets for the neighboring inclusions are used to perform the slicing operation. Being $a d$-hoc functions, the local ${ }^{I} D N_{k i}$ functions are constrained to be equal to their global counterparts inside ${ }^{I} \Theta_{i}$ and $C^{1}$ continuous across the boundary $\Phi_{\Theta i}$. The values of the local function inside ${ }^{I} \Theta_{i}$ are intended to yield the same results as their global counterparts while the values in ${ }^{O} \Theta_{i}$ do not matter as they will be sliced off. However, in order to enable proper contouring, the surface has to be smooth on $\Phi_{\Theta i}$.

The values of the local ${ }^{I} O P_{i}$ can be obtained by a simple reorganization of the $D N_{k}$ functions, which consist of assemblies of patches coming from $D S_{i}$. This reorganization can be achieved keeping in mind the aforementioned constraints necessary to construct ${ }^{I} O P_{i}$ :

$$
\begin{gathered}
{ }^{I} D N_{1 i}=D S_{i}(\mathbf{x}), \\
{ }^{I} D N_{2 i}=\left\{\begin{array}{l}
D N_{2}(\mathbf{x}) \text { where } N N_{2}(\mathbf{x}) \neq i, \\
D N_{1}(\mathbf{x}) \text { where } N N_{2}(\mathbf{x})=i,
\end{array}\right. \\
{ }^{I} D N_{3 i}(\mathbf{x})=D N_{3}(\mathbf{x}) .
\end{gathered}
$$

Eqs. 21a and 21b are illustrated in Figures $17 \mathrm{a}$ and $17 \mathrm{~b}$, respectively. Figure 17t illustrates the extraction of the triangular slices using "local" $t$ level sets of adjacent inclusions. Equations $\sqrt{21 \mathrm{c}}$ can be used as $D N_{3}$ is already $C^{1}$ continuous across $\Phi_{\Theta i}$. Further discussions on the extraction of these functions can be found in appendix of [41. To avoid the memory requirements linked to the storage of the "local" functions of all inclusions, these functions can be constructed in a simple and efficient manner using indexed operations whenever a particular surface from an inclusion needs to be treated, even for large RVEs with a large number of inclusions.

It should be noted that the operators necessary for introducing ligament curvature 


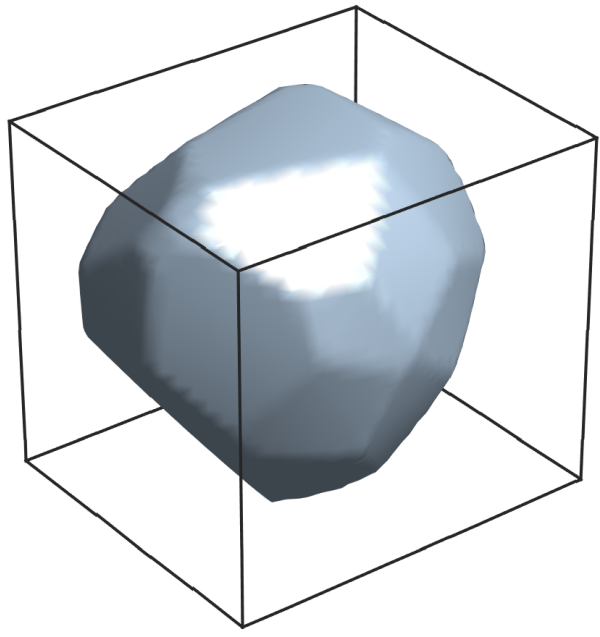

(a)

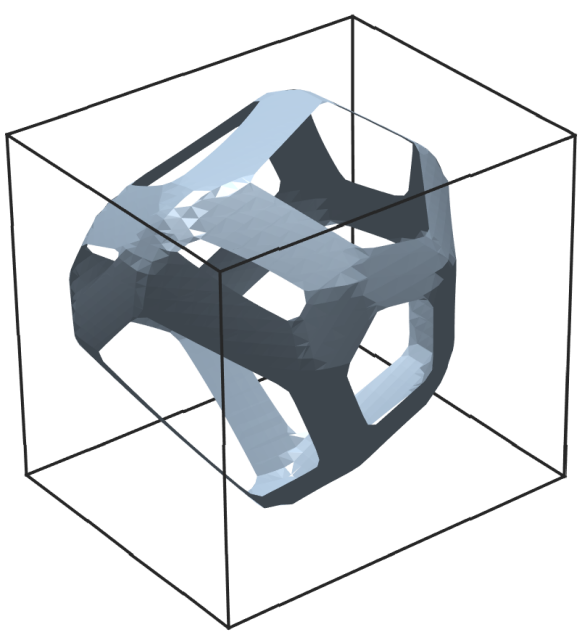

(b)

Figure 16: Illustration of multiple level sets approach on a single isolated cell corresponding to an inclusion; (a) Surface resulting of the contouring of the "local" level set function ${ }^{I} O_{P i}$, and (b) surface after slicing by the "local" level set functions of the neighboring inclusions.

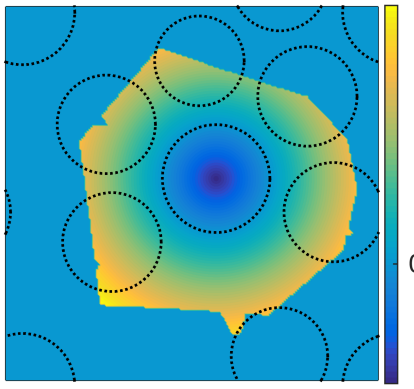

(a)

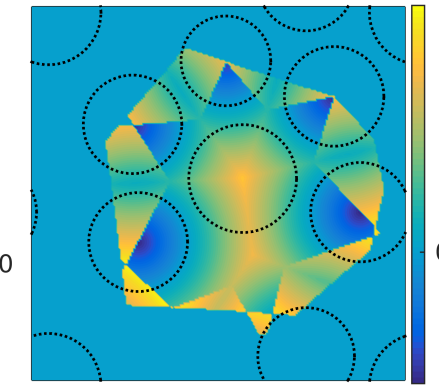

(b)

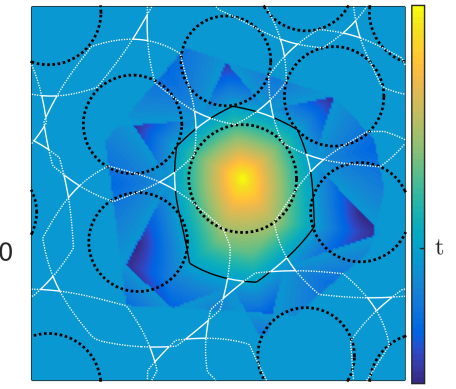

(c)

Figure 17: (a) Modified "local" I $D N_{1 i}$ function, and (b) modified "local" ${ }^{I} D N_{2 i}$ function, for inclusion $i$. The difference with the global functions plotted in Fig 5 can be seen by comparison. (c) ${ }^{I} O P_{i}$ function for inclusion $i$ is plotted with the $t$ level set drawn in black, while those of the immediate neighbors are drawn in dotted white. The triangular slices that form the part of the global $t$ level set have also been plotted in bold white for reference. 
and strut cross-section variation can be built globally and used in a straight-forward manner along with the local ${ }^{I} D N_{k} i$ functions. Also, a significant computation cost can be saved by limiting the computation domain to a band enclosing the surface to extract, which can be identified with the help of the $N N_{k}$ functions:

$$
\begin{gathered}
D O I_{i} \equiv\left(\left(N N_{1}(\mathbf{x})=i\right)\left|\left(N N_{2}(\mathbf{x})=i\right)\right|\left(N N_{3}(\mathbf{x})=i\right)\right) \\
\&\left(\left(O_{V}(\mathbf{x})-t>-e\right) \&\left(O_{V}(\mathbf{x})-t<e\right)\right),
\end{gathered}
$$

where $e=t / 2$ and is at least twice the discretization size of the domain. Figure 15 illustrates the extraction of an RVE by the help of multiple level sets with the desired features.

\section{Finite element mesh generation}

For use in a computational simulation, it is necessary to generate the volume discretization of the geometries obtained from the level set functions discussed before. For this purpose, a simple mesh generator is used that takes into account the slicing operations while giving a consistent discretization along the sharp edges. The tool developed in 51 is therefore used, where dynamic node repositioning based on level set functions is used to build high quality conforming meshes using the strategy developed in 42. A similar strategy has been used in [52] to generate textile reinforced composites RVEs.

The sharp edges can be extracted from the intersecting surfaces extracted from the definition of ${ }^{I} O_{P}$ of two adjacent inclusions using any of the standard Boolean operations on surfaces, like the triangle/triangle intersection test routine [53], or the Boolean operation tool on 3D polyhedra [54. For any two adjacent inclusions, the surface extracted using the intersection approach is a combination of the two sliced surfaces sharing a common curve representing the sharp edge (Figures $18 \mathrm{a}$ and $\mathrm{b}$ ).

The standard approach to extract triangulated surfaces from discrete points in a grid is to use the isosurface technique based on the marching cube method developed by Loreson and Cline [55] according to a pre-specified variable target size function, $h(\mathbf{x})$. The triangulated surfaces constructed can then be used as a constraint towards tetrahedral mesh generation by 3D Delaunay Triangulation. The Constrained Delaunay Triangulation (CDT [56) module implemented in the software Tetgen [57 is used to obtain a conformal mesh for which the constraint ensures that the output contains the faces of surfaces discretizing the material boundaries while preventing elements to span across material boundary interfaces.

However, this approach, when used directly, leads to a notable presence of ill-shaped faces (too small, too narrow, etc) resulting from the initial surfaces extracted from the isosurface technique. It is therefore unfit for a proper use in finite element computations. To overcome this, a mesh quality optimization process is applied on the mesh. The common curves representing the sharp edges are re-interpolated initially based on $h(\mathbf{x})$ (Figure 18) and then the surface elements of the isosurfaces are re-triangulated keeping this re-interpolated curve of the sharp edge fixed (Figure 19).

The ideal situation of a high quality mesh, i.e., the presence of only quasi-regular tetrahedra with edge lengths $l^{e}$ equal to the value of the targeted element size map $h(\mathbf{x})$ at corresponding edge mid points $\mathbf{x}_{e}$, cannot be reached exactly for complex RVEs and 
varying element sizes. However, configurations (or node positions) can be found that minimize the magnitude of the difference,

$$
F^{e}=l^{e}-h\left(\mathbf{x}_{e}\right),
$$

over the mesh, which helps obtaining optimal element quality for a given triangulation. The methodology for mesh optimization follows an original idea used in 42] where an analogy with the equilibrium configuration of a 3D truss of elastic bars is applied to the optimization of the edges of the tetrahedral mesh. The bars of this auxiliary truss are subjected to a force $\mathbf{f}^{e}$ acting along the bars, proportional to $F^{e}$ in Eq. (23). These forces cancel out at the nodes by mechanical equilibrium. This procedure is equivalent to the application of an internal force field steering the lengths of the truss bars towards $h(\mathbf{x})$. A fictitious resultant force $\mathbf{f}\left(\mathbf{x}_{n}\right)$ is defined that acts on all nodes positions that need to be optimized. This force is simply the vectorial sum of $\mathbf{f}^{e}$ acting on all such nodes and thus, for a given triangulation, equilibrium requires

$$
\mathbf{f}\left(\mathbf{x}_{n}\right)=0 .
$$

This condition corresponds to the optimal node positions $\mathbf{x}_{n}$ as the result of a fictitious mechanical equilibrium. A fictitious time dependency can be introduced to solve this equations system,

$$
\frac{\mathrm{d} \mathbf{x}_{n}(t)}{\mathrm{d} t}=\mathbf{f}\left(\mathbf{x}_{n}\right),
$$

along with a forward first order Euler time integration scheme,

$$
\mathbf{x}_{n}(t+\mathrm{d} t)=\mathbf{x}_{n}(t)+\mathrm{d} t \mathbf{f}\left(\mathbf{x}_{n}\right),
$$

to reach a stationary state from the base mesh based on a defined tolerance.

When meshing and optimizing the three different outer surfaces of the foam struts, it is important to ensure that the nodes located on the common sharp edges remain fixed. Subsequently, when meshing the strut volumes, surface nodes are kept fixed using the already stored level set information. The nodes lying on the RVE faces are treated to ensure that they remain on the faces and satisfy any periodicity conditions applied. In practice, to ensure robustness and versatility, the optimization process is thus applied on the extracted sliced isosurfaces and the RVE faces first followed by CDT, with the optimized surface acting as the constraint. This step of global optimization leaves the already optimized nodes untouched. Note that a similar force-based approach could also be implemented to optimize the common sharp edges instead of a simple re-interpolation. Figure 20 illustrates a portion of the RVE before and after surface optimization. Figure 21 illustrates a portion of the RVE after the CDT process and a sectional view of the tetrahedrals.

\section{Morphology Quantification of the resulting RVEs}

In this section, we quantify some of the important morphological parameters of the RVEs generated based on the DN-RSA. We first look at the properties that are directly related to the packing generation, and subsequently compare the properties of the meshed 


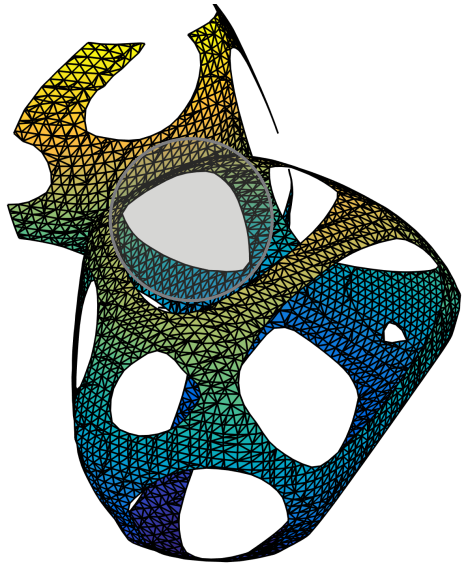

(a)

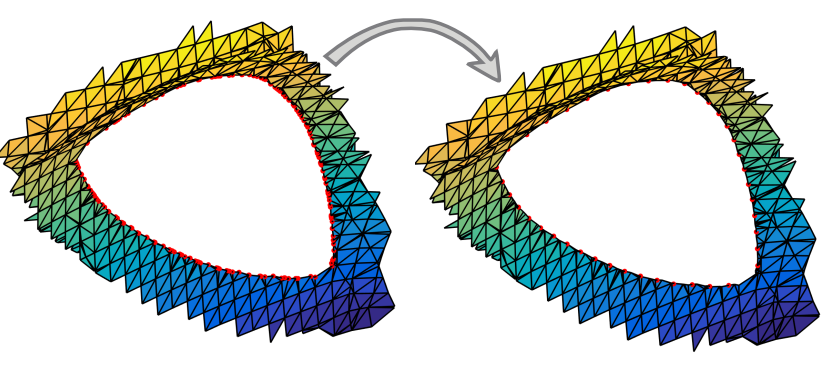

(b)

Figure 18: (a) Common curve extracted from the intersection of the surfaces obtained from ${ }^{I} O_{P}$ function of two adjacent inclusions. (b) The re-meshing of the common curve of intersection using a piecewise cubic Hermite polynomial interpolation. The red dots represent the nodes being remeshed.

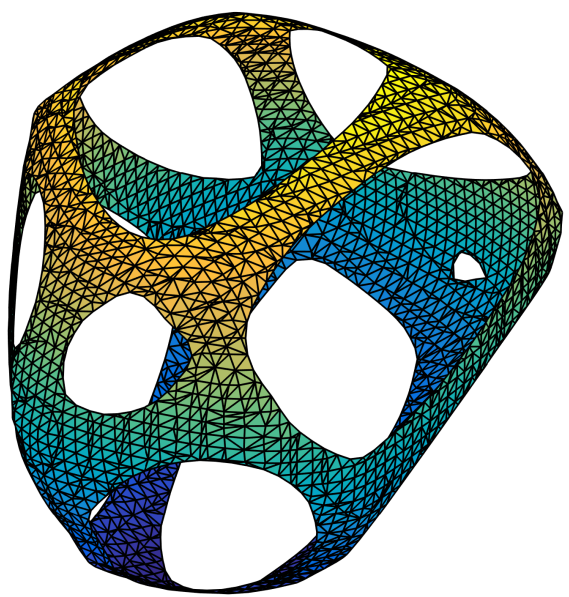

(a)

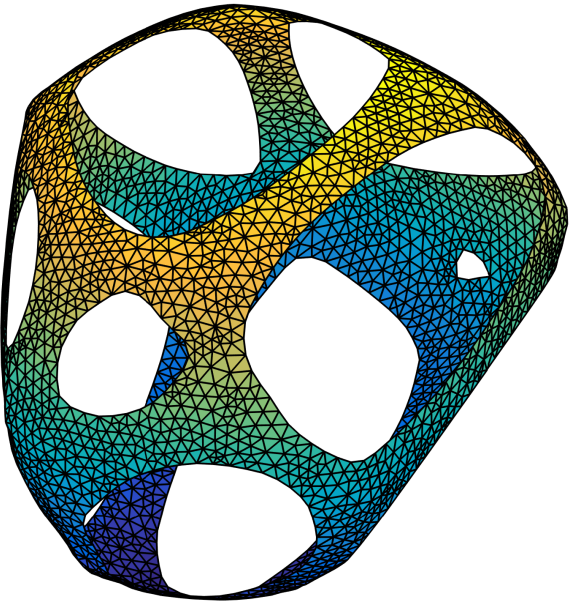

(b)

Figure 19: (a) Surface extracted from ${ }^{I} O_{P}$, sliced and common curves re-interpolated. (b) Surface after optimization by force based tensioning scheme. 


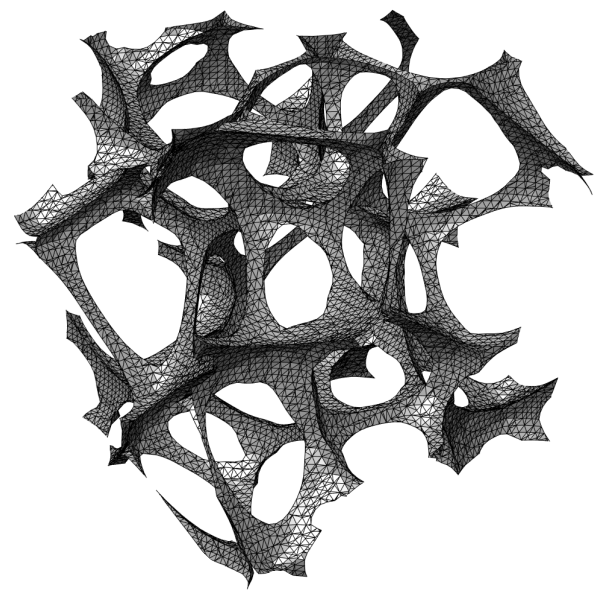

(a)

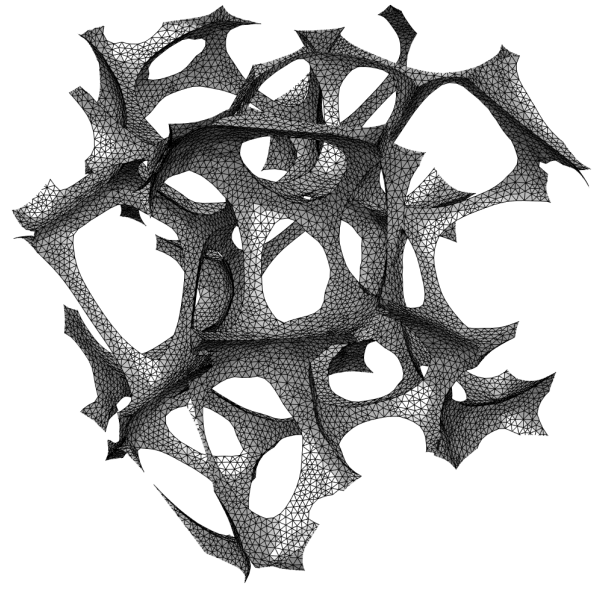

(b)

Figure 20: Sectional representation of an RVE generated by DN-RSA (a) before, and (b) after surface mesh optimization process.

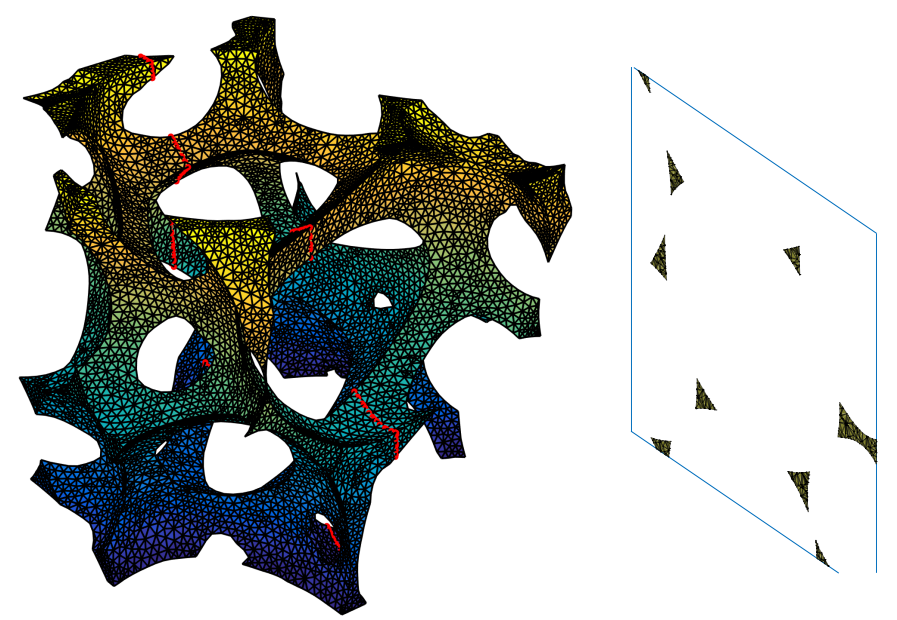

Figure 21: Conformed 3D mesh made of tetrahedrals after CDT process using Tetgen. 
RVEs after the implementation of tools that use distance functions to vary the strut morphology.

\subsection{Validation of the Laguerre parameters}

The quantification of the morphological parameters is a necessary step in order to compare the properties of the resulting RVE geometry with real samples. Studies have been conducted to fit Laguerre tessellations to foam microstructures, where it was observed that properties like cell volume, surface area, mean width and number of facets per cell are sufficient to fit the models to real sample [32. In [31, observations of Laguerre tessellations generated by random packing of spheres with lognormal and gamma distributed volumes have been presented with a coefficient of variation (CV, ratio of standard deviation and the mean of the volume distribution of the initial sphere packing). These observations show that for lower values of CV $(<0.5)$, the chosen volume distribution and the sphere packing do not have a significant effect on the models generated. Since the aim of the procedure is to be used in multi-scale simulations, and commercially available metallic foams tend to have low $\mathrm{CV}(<0.1)$ [4], the present contribution is limited to lognormal distributions.

\subsubsection{Methodologies}

Two approaches can be envisioned to evaluate the parameters under scrutiny.

1. A "classical" approach makes use of the triangulated surfaces extracted from the level sets by contouring. Generally this approach can be used once the final mesh is generated and gives accurate values even for coarse discretizations. Apart from being able to calculate volumes and surfaces, by using the slicing functions discussed in the previous sections, isolated tessellation volumes can be extracted and their face, edge and node data can be characterized.

2. An "implicit" approach directly makes use of the functions already computed, mainly $N N_{k}, D N_{k}, O_{V}$ and $O_{P}$. It has to be pointed out that the precision and accuracy of the results obtained in this approach largely depend on the size of the discrete grid used for the evaluation of these functions. Parameters that depend on the tessellation, like face-by-cell count and edge-by-face count can be calculated by counting the number of direct neighbors stored in the $N N_{k}$ functions. The tessellation volumes and overall foam/coating volumes can be calculated by counting grid points with relevant values of the level set functions, and dividing the obtained number by the total number of grid points.

An alternative way to quantify the morphology externally consists of using the convex hull approach with which, once the spheres packing is obtained, the tessellations can be simulated by constructing the convex hull of the centers of the spheres. The properties can then be extracted using QHull software package [58, as detailed in 31 to study the properties of Laguerre tessellations. It should be noted here that the QHull package is extremely precise in modeling the tessellations. As a result, it is able to capture even the smallest faces and edges regardless of the discretization used. In the limit of a vanishing discretization size, this should also be the case for the "implicit" approach. However, in practice, with finite discretization grids, situations will arise in which two very close edges (or nodes) will be seen as a single one, shared by 4 or more faces (or 5 or more 


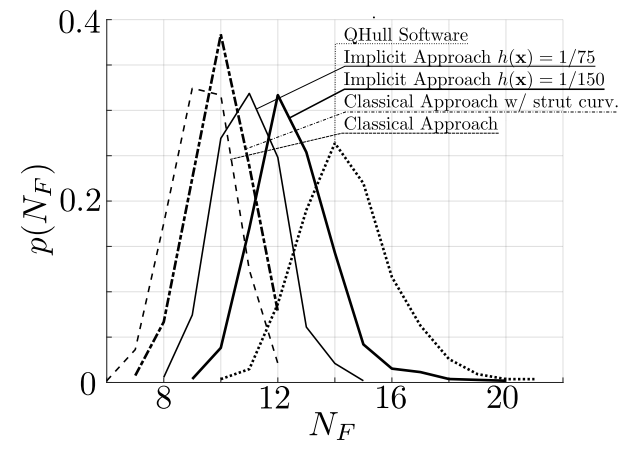

(a)

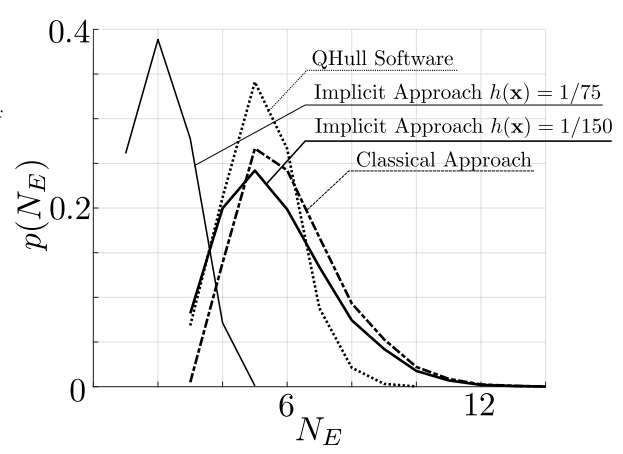

(b)

Figure 22: (a) Comparison of the occurrence of the number of faces per cell using various approaches for the same RVE with free boundary. (b) Comparison of the occurrence of the number of edges per face using various approaches for another RVE with free boundary. Sphere packing has been generated with $C V=0.07$ and the number of spheres 750 .

edges). Also, the geometries extracted from the "classical" approach will have already incorporated a finite edge thickness, resulting in further reduction of the number of faces per cell.

\subsubsection{Number of faces per cell and number of edges per face}

Figure 22a shows a comparison between various approaches to compute the number of faces per cell for an RVE constructed using free or non-constrained boundary conditions. Out of the 750 inclusions initially generated, only 400 remain inside the domain after minus sampling operation as discussed in Section 2.1. The effect of refining the discretization grid for the function evaluations can be noticed in the increase in the mean number of faces captured using the "implicit" approach, with the curve shifting towards the data obtained from QHull package as the number of grid points increases. It is also interesting to note that the "classical" method captures a lower number of faces per cell as explained before. Similarly, Figure 22b compares the effect of the different approaches for calculating the number of edges per face. As a value for comparison for these quantities, the mean number of faces per cell values ranges from 12 to 14 for a mono-dispersed open foam (with very low CV), as analyzed by CT scans according to various works that can be found in literature [59. Similarly, the number of edges per face ranges between 5 and 5.5. Figure 23 illustrates the effect of a variation of CV on two sample properties, i.e. the number of faces per cell and the coefficient of variation of the volumes of the resulting cells of the tessellation in comparison with that of the initial packing sphere volumes. These values are in sync with the values that were obtained in 31.

\subsubsection{Edge length distribution}

The "implicit" approach provides a very straightforward approach to compute the edge length distribution of an RVE. By making use of operator (13), a distribution of the edge length can indeed be obtained. In the "classical" approach based on triangulated surfaces, the introduction of a finite thickness for the struts results in very small faces and edges being absorbed and represented as very thick struts. This factor cannot be 


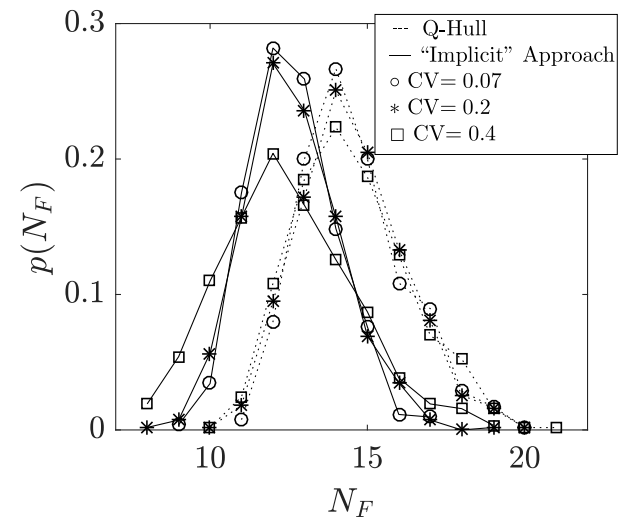

(a)

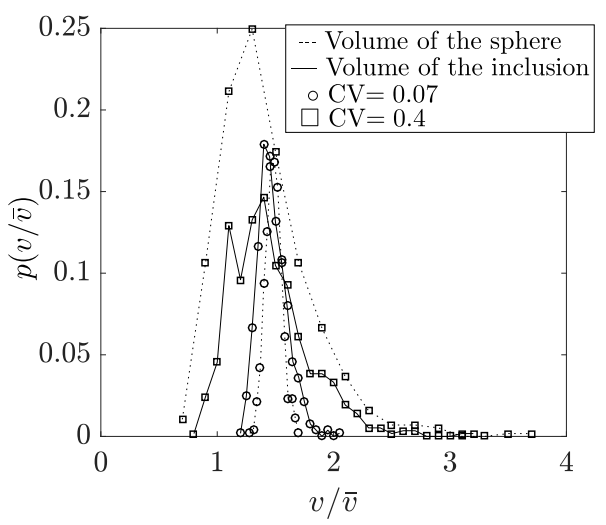

(b)

Figure 23: (a) Variation of number of faces per cell with change in CV; (b) Comparison between the normalized volumes of the spheres in the initial packing and the normalized volumes of the resulting inclusions with variations in $\mathrm{CV}$.

included in the "implicit" approach directly. A relation graph between the cells, faces, edges and nodes can be built, and a comparison between the "implicit" and "classical" approaches based on this graph can help evaluating the exact number of struts in the generated RVE. Many studies, like 28, 60, utilize structural beams with uniform crosssection to represent the struts in the RVE and introduce a control on the minimum strut length by collapsing struts shorter than a tolerance parameter to a single node, in order to decrease the computation time. This is not necessary when constructing RVEs using distance functions, as the struts are implicitly meshed based on the extracted isosurfaces before generating a volume mesh. The influence of relatively short struts is therefore expected to have almost no effect on the mechanical behavior of the unit cell. This technique is however helpful in getting a rough estimate of the strut length distribution. In general, the Q-Hull approach generates the data of the tessellations in the form of a list of polygons that form the faces of the individual cells, which can then be compared with the relation graph to identify such short struts and also the resultant closed faces. One can then merge all the nodes that are close to each other for these identified faces and obtain the data about an approximate strut length distribution. Figure24illustrates the variation of the normalized edge lengths with respect to changes in CV as computed by Q-Hull, which shows trends similar to those found in [36] for Laguerre tessellations. The distributions become more Gaussian when comparing the lengths with the relation graph in which all the small edges and faces are absorbed by the implementation of a thickness parameter ( $t$, in these simulations, is chosen such that the ratio between the pore diameter and the strut thickness is approximately $6: 1$ ).

\subsubsection{Conclusions on the statistical distribution of RVE properties}

The study of the aforementioned properties of the tessellations developed using distance functions of packings generated by DN-RSA shows that they sufficiently match the ones developed by existing tools in literature. These studies have also shown that, for sufficiently low CV values, the models fit quite well with real samples, i.e. the random- 


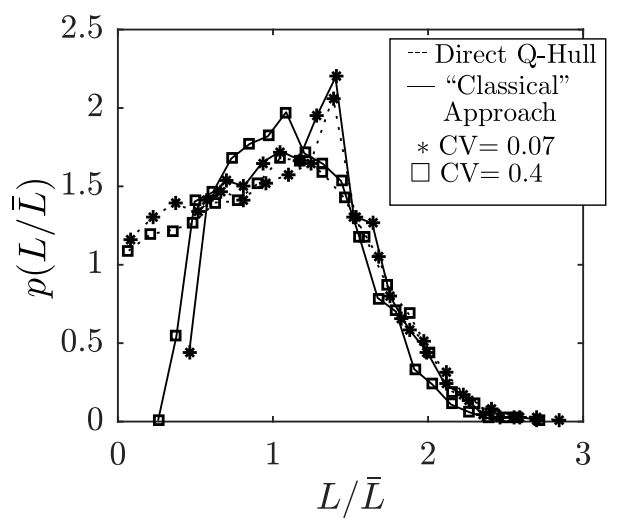

Figure 24: Normalized edge length distribution of an RVE with free boundary.

ness generated by using Laguerre tessellations matched the natural randomness of open foams with mono-dispersed inclusions primarily considered in industrial applications.

To model such foam samples, it is sufficient to consider a large number of closely packed spheres so that in a non-periodic RVE, even after the use of minus-sampling, as explained in Section 2.1, there will be a sufficient number of inclusions that will allow properly reproducing the randomness of the foam sample. Table 1 analyses the relation between the average size of the inclusions, represented here in terms of the number of inclusions in the domain, and the maximum permissible unit length for minus sampling. It can be seen that with an increase in the number of inclusions in the RVE, more inclusions can be retained inside the reconstructed domain. However, it also means that non-periodic RVEs need to be larger than the periodic RVEs.

An approach to model foam structures based on the computation of the equilibrium microstructure of soap froth was presented in [12] using Brakke's Surface Evolver software [61, 14. The tool starts with a random packing of spheres and uses the principle of minimization of surface energy that shapes the "liquid" into Plateau borders. The resulting structure is a periodic and isotropic RVE with a Gaussian-like strut length distribution. Such a process requires an important computational effort, as a large number of facets are required to discretize the Plateau borders. Also, the skeletal or a stick-figure model is extracted and the ligaments are simply modeled as shear deformable beams with the cross-section geometry based on the measured area distribution of the foam sample. This requires a further material volume correction at the nodes of the ligaments to account for excess material due to beam overlap. It was shown that extracting the seed points of a possible Laguerre tessellation based on the intersection nodes of a given foam sample produces tessellations that are similar in terms of morphological properties with those of simulated foams generated using Surface Evolver 62. The use of these seed points in the DN-RSA algorithm directly instead of a distribution of radius to generate an open foam RVE would be an interesting further development. 
Table 1: Study of the effect of minus sampling on the number of inclusions that can be used for the extraction of an open cell foam RVE.

\begin{tabular}{l|l|l}
\hline $\begin{array}{l}\text { Total spheres in the initial } \\
\text { packing }\end{array}$ & $\begin{array}{l}\text { Inclusions remaining after } \\
\text { minus sampling }\end{array}$ & $\begin{array}{l}\text { Maximum permissible unit } \\
\text { length for minus sampling } \\
\text { (\% of initial RVE length) }\end{array}$ \\
\hline 331 & 122 & $0.7 \%$ \\
438 & 172 & $0.74 \%$ \\
483 & 229 & $0.78 \%$ \\
564 & 277 & $0.8 \%$ \\
690 & 331 & $0.81 \%$ \\
751 & 402 & $0.82 \%$ \\
\hline
\end{tabular}

\subsection{Verification of morphology variation}

\subsubsection{Foam density}

As in the previous section, the morphological variations obtained by the "postprocessing", or the extraction of the RVE geometry through the combination of distance functions can also be investigated using both the "implicit" and the "classical" approaches. For a sufficiently mono-dispersed foam, the developed model can mimic the changes due to the variations in ppi by introducing variations in the strut morphology without the need to build models with a large number of inclusions to achieve the same results. As can be seen in [37, 43, the strut cross-section changes from a plateau-border configuration to a circular shape as the ppi value increases. This can be explained by the solidification of the base PU foam before reaching equilibrium at lower ranges of the sample dimensions. This variation can also be seen in the excess accumulation of material at mid-section of the struts in higher ppi foam samples.

The density of the foam can be computed "implicitly" by counting the grid points with relevant values of the $O_{P}$ function. In the "classical" approach this can be achieved by calculating the sum of the volumes of the tetrahedral elements in the RVE mesh. To achieve variations in the ppi value, and thus the density in an RVE with unit dimensions, the variations in the strut cross-section play an important role. Table 2 shows that the difference in the computed densities between the two approaches remains rather small. Since the "Implicit" calculation can be done as a post-processing operation of DN-RSA without extracting the mesh, the relative density can be computed initially, and then adjusted using the quantities that influence the strut cross-section variation and thickness to obtain an RVE with a ppi value close to the real samples. Figure 25 compares the effect of a strut cross-section variation and of a changing thickness parameter on the overall porosity computed by the "Implicit" approach.

Based on the manufacturer's description or on previous studies on foam samples using CT scans, it is possible to build a link between the ppi values and the average cross-sectional area of the struts of the foam at mid-sections. Based on [12, 37, 43, the values of $c_{1}$ and $c_{2}$ in Eq. (11) as well as the control on concavity, $k_{c}$ in Eq. $(20)$, can be assigned. These values have to be calculated on a case by case basis. However, a general data can be prepared, with variations in the values that depend on the manufacturing 
Table 2: Comparison of relative density calculated by the two approaches for an RVE with free boundary. Density variations was achieved by modifying the variables influencing the strut cross-section.

\begin{tabular}{ccc}
\hline "Classical" Approach & "Implicit" Approach & Difference \\
\hline 0.0216 & 0.0205 & $5.09 \%$ \\
0.0647 & 0.0586 & $9.42 \%$ \\
0.0641 & 0.0614 & $4.21 \%$ \\
0.0652 & 0.0604 & $7.36 \%$ \\
\hline
\end{tabular}

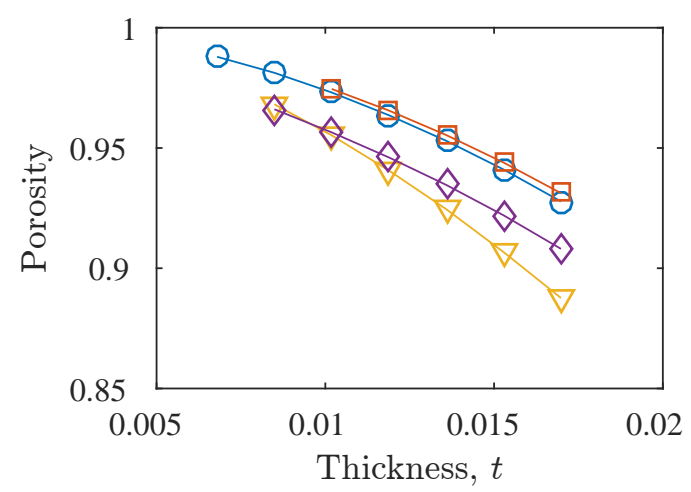

Figure 25: Variation of the RVE porosity with strut thickness and cross-section variations in Eqs 11 and 20; Legend - $-: c_{1}=20, \square: c_{1}=60$ denoting thinner strut cross-section at mid strut, $\triangle$ : $c_{1}=3$ denoting almost uniform cross-section, and $\diamond: c_{1}=20, k_{c}=-0.5$ with convexity in the strut cross-section.

process used for the foam and the degree of inclusion volume dispersion.

\subsubsection{Struts cross-section}

Individual sample struts can be analyzed using the "implicit" approach, by identifying the four nearest inclusions of the struts, identifying the grid points of the strut by matching them with respective $N N_{k}$ values and calculating the value of $O_{P}$ at these grid points. Using the "classical" approach, the tetrahedra elements that form the struts can be identified due to their proximity to the grid points identified in the "implicit" approach. The discretization grid however needs to be very small to have a good approximation of the analysis of a strut using the "implicit" approach. It can also be used as a starting point to analyze the struts through the "classical" approach. Figure 26 illustrates a strut extracted from an RVE and the variation of the cross-sections along the axis. It is to be noted that the ends of the struts are already a part of the node and thus the sections in these parts are not triangular anymore. This also ensures that material accumulation due to overlapping struts does not happen since the extracted surface is a direct result of the distance function. Figure 27 compares the variations in the concavity-convexity that can be obtained by varying the concavity parameter $k_{c}$. The variation of the relative area along the axis of 5 sample struts extracted from an RVE 


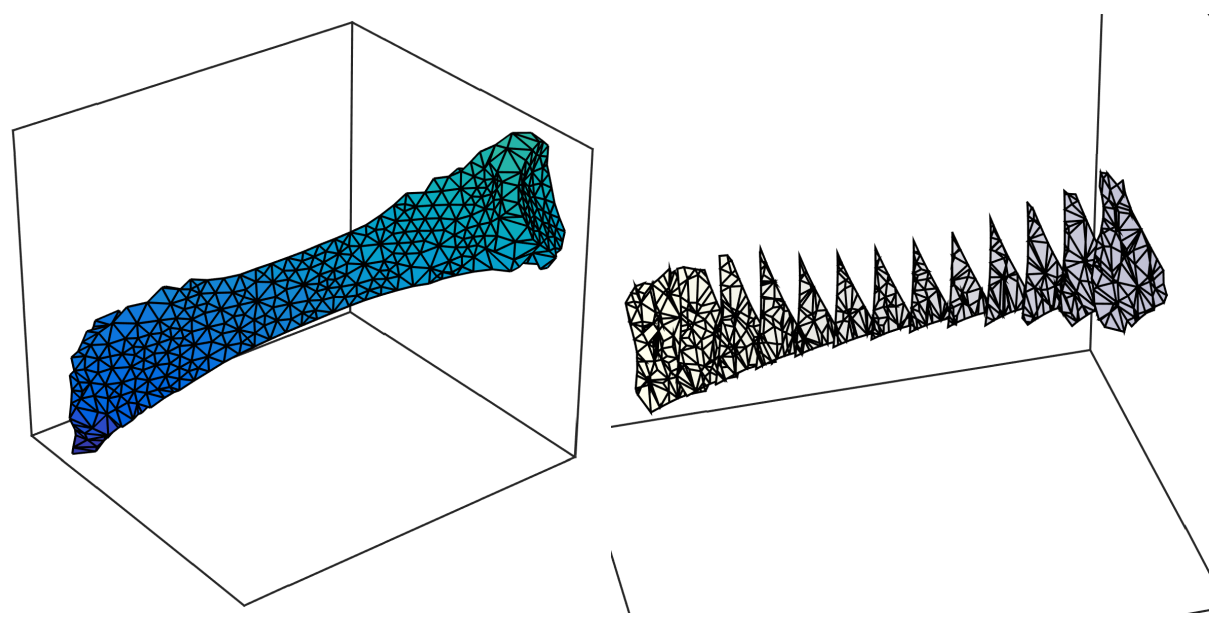

Figure 26: A single sample strut taken from an RVE displaying the behavior of a 20 ppi foam and the cross-section variation comparison at different axial positions.

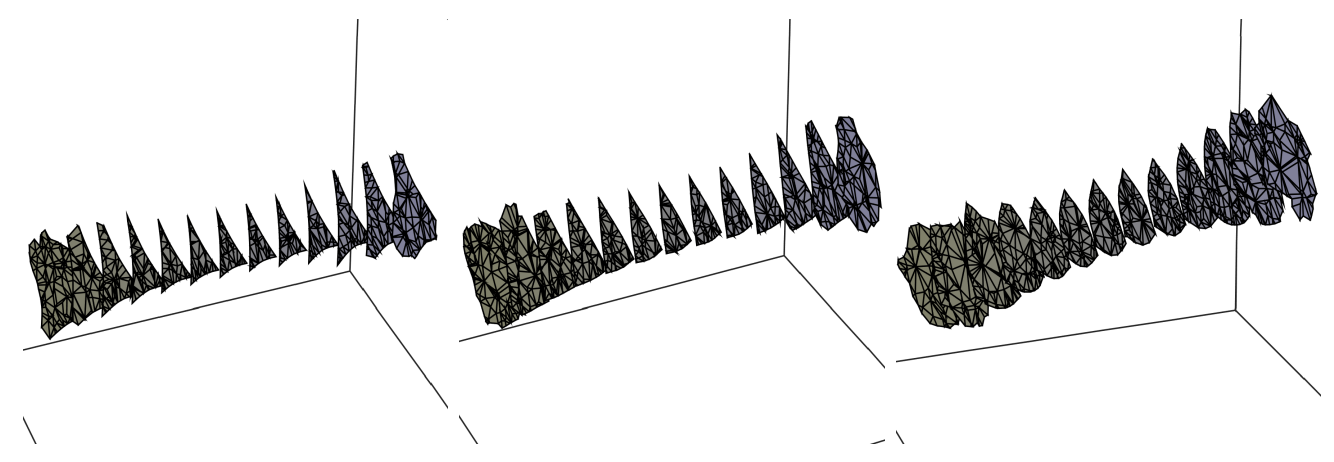

$\begin{array}{lll}\text { (a) } k_{c}=0.2 & \text { (b) } k_{c}=-0.2 & \text { (c) } k_{c}=-0.5\end{array}$

Figure 27: The cross-section variation comparison by using different concavity paramater, $k_{c}$, at midspan of the struts.

is illustrated in Figure 28 in which it can be seen that, when compared to the relative area variation of a strut taken from a real foam, the RVE mimics the strut cross-section faithfully.

\subsubsection{Generation of targeted morphology}

From values reported in Table 3 , it can be seen that the standard values for porosity with varying ppi are quite dispersed statistically. However, a linear correlation can be used for the sake of simplicity. Since the samples in these studies are mono-dispersed open-cell Aluminium foams, a closed packing of nearly mono-dispersed spheres can be generated using DN-RSA. Once the packing of spheres is generated, based on individual tessellation volumes, the mean characteristic length of the inclusions can be obtained. This value can be combined with the thickness to radius ratio obtained in Table 3 and the respective values of coefficient $c_{1}, c_{2}$ and $k_{c}$ depending on the ppi to generate an 


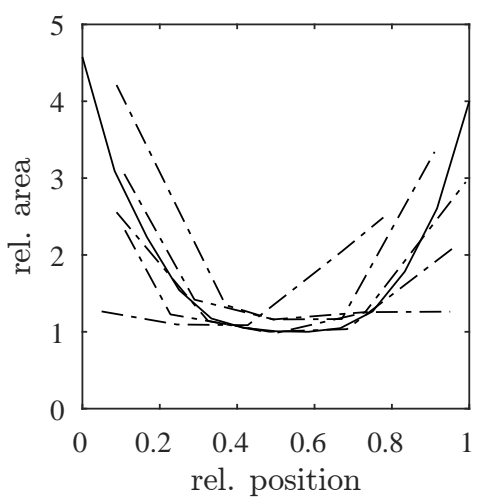

Figure 28: A comparison of strut cross-section area with experimental data (in dotted lines, re-sketched from 37] for 20 ppi foams), while the bold line depicts the variation for the strut displayed in Figure 26

Table 3: Approximate combination of the study of variation of porosity and strut thickness to inclusion radius ratio with ppi based on data on samples of Aluminium foam by ERG and Celltec materials as observed in 43 and 37 .

\begin{tabular}{|c|c|c|c|c|c|c|}
\hline & \multicolumn{2}{|c|}{ Literature experimental values } & \multicolumn{4}{|c|}{ Values used in DN-RSA } \\
\hline ppi & $\begin{array}{l}\text { Porosity } \\
\text { in } \% 37\end{array}$ & $\begin{array}{l}\text { Average strut thickness } \\
\text { to average inclusion } \\
\text { radius ratio } 43\end{array}$ & $\begin{array}{l}\text { Values } \\
\text { of }\left[\begin{array}{ll}c_{1} & c_{2}\end{array}\right]\end{array}$ & $\begin{array}{l}\text { Value } \\
\text { of } k_{c}\end{array}$ & $\begin{array}{l}\text { Average strut thickness to } \\
\text { average inclusion radius } \\
\text { ratio for } t \text { in Eq. }\end{array}$ & $\begin{array}{c}\text { Obtained } \\
\text { porosity }\end{array}$ \\
\hline 5 & - & 0.2310 & - & - & - & - \\
\hline 10 & 0.942 & 0.1945 & {$\left[\begin{array}{ll}50 & 1\end{array}\right]$} & 0 & 0.2050 & 0.9418 \\
\hline 20 & 0.937 & 0.1983 & {$\left[\begin{array}{ll}25 & 1\end{array}\right]$} & -0.05 & 0.2 & 0.9388 \\
\hline 30 & 0.916 & - & {$\left[\begin{array}{ll}10 & 1\end{array}\right]$} & -0.2 & 0.198 & 0.92 \\
\hline 40 & - & 0.1878 & - & - & - & - \\
\hline
\end{tabular}

open-foam RVE. Simple interpolation can be used for values that lie in between. The final column of Table 3 shows that the porosity values obtained match to a good extent, while maintaining the morphological similarity of the RVE foam with real foam samples.

Figure 29 shows the implementation of the anisotropy parameter (in percentage) along $\mathrm{X}$ axis on the packing to extract anisotropic morphologies from a single DN-RSA packing as described in Section 3.4. This parameter is particularly helpful in implicitly implementing the anisotropy that are observed in actual foams due to the solidification of cast metal in the rise direction.

\section{Finite element modeling of the compressive response of foams}

In this section, a finite element simulation is conducted to demonstrate the ability of the RVE generation procedure to reproduce the structural properties of open foam. First, a sample micro-cell is generated based on morphological parameters of an open 


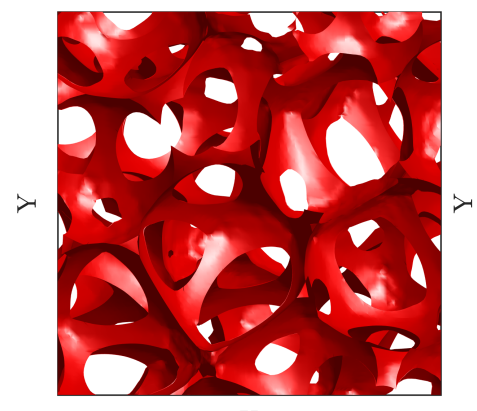

$\mathrm{X}$

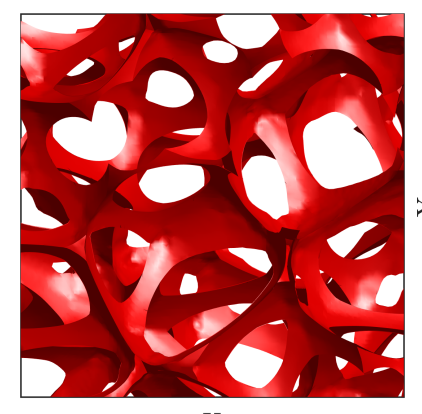

$\mathrm{X}$

(b) $25 \%$ anisotropy

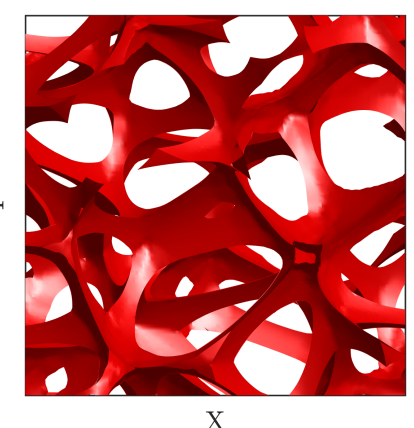

(c) $50 \%$ anisotropy

Figure 29: Implementation of anisotropy variation on a foam generated from a single DN-RSA packing along $\mathrm{X}$ axis.

foam sample available from literature [37]. Material parameters identified in [63] are used for the struts constitutive model. A single pore will be identified initially and a compression test will be simulated. The results will be compared with that reported in 63. Then an entire generated RVE is computed under a uniaxial compression, using the finite element procedures proposed in [49, 64. Finally, a comparison is built between the model predictions and the experimental data provided in 37.

\subsection{Generation of the RVE and material properties}

An RVE of open-foam is prepared which mimics the morphology of Aluminum foam investigated under uniaxial compression in [37. The generation uses the DN-RSA procedure with non-periodic conditions and a mono-dispersed volumes of an initial sphere packing with around 125 spheres in the RVE. After minus-sampling, around 25 inclusions remain in the RVE. The radii of the spheres in the packing are randomly chosen from a logarithmic distribution with a CV of 0.07 and a mean radius of $0.125 \mathrm{~mm}$. The strut morphology is varied to match the ppi of the foam with which it is compared. With the help of Figure 25 and Table 3, appropriate values can be selected to mimic a foam with ppi between 20 and 30 with $c_{1}=20, c_{2}=1$ and $k_{c}=-0.1$. Additionally, an anisotropy in the pore geometry is also introduced during the minus-sampling along the direction in which the uniaxial test is conducted to mimic the anisotropy present along the rise direction during the manufacturing phase. In this case, based on the experimental observations in [37, we propose an anisotropy coefficient of 1.4 along the rise direction.

For illustration, the struts obey a linear hardening hyperelastic-based J2-elastoplastic material law formulated in large strains (Appendix A of [49]), with the isotropic hardening law

$$
\sigma_{y}^{0}\left(\bar{\varepsilon}^{p l}\right)=\sigma_{0}+H_{i s o} \bar{\varepsilon}^{p l} .
$$

Standard bulk material properties of AlSi7Mg0.3 alloy are in general much higher than those identified in experimental tests on foamed materials 63. using experimental forcedisplacement diagrams. This is due to the differences in the foams manufacturing process that cause changes in grains structure and texture. In [63], the authors used an inverse 


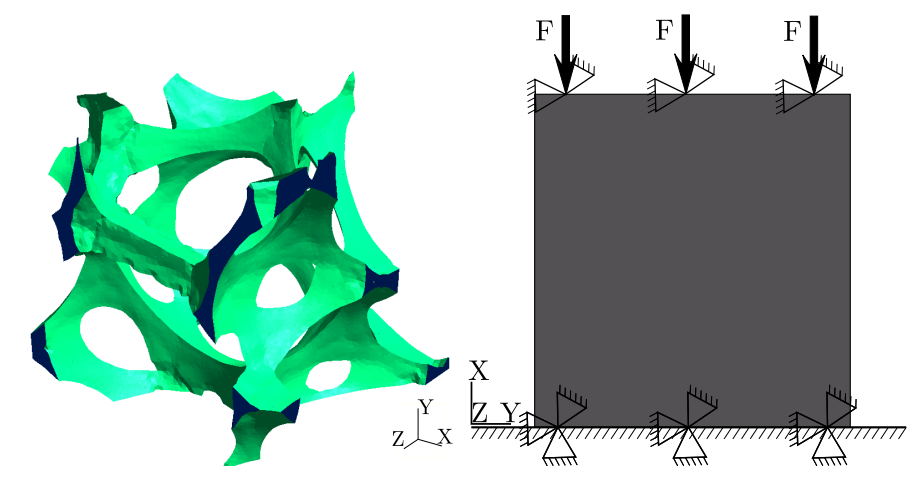

(a)

(b)

Figure 30: (a) Sample pore extracted by DN-RSA, and (b) the boundary conditions used in the simulations.

identification of the struts material properties from the compression test based on a single pore, which served as a geometric model of a FE simulation. We use the average identified values of $E=3968.12 \mathrm{MPa}, \sigma^{0}=46.35 \mathrm{MPa}$, and $H_{i s o}=214.61 \mathrm{MPa}$.

\subsection{Numerical Simulations}

\section{Single pore}

In 63], 5 sample pores were extracted from an open foam sample with an average size of each pore around $6 \times 6 \times 6 \mathrm{~mm}^{3}$. These were subjected to a compression test with the force-displacement response recorded. These samples were poured in Wood's metal to restrict strut movement and rotation as the samples were very small.

Since the samples being tested in the literature are from the same manufacturer as in [37, some sample single pores have been extracted from an RVE, and is scaled such that the average pore length for a $20 \mathrm{ppi}$ foam is achieved. To mimic the compression test, the bottom faces along the preferential anisotropy direction in the samples are restricted in all directions. The top faces are restricted along the directions perpendicular to the loading direction (Figure 30 .

In Figure 31, sample pore geometries taken from the RVE have been subjected to a uniaxial compression test. The yellow band represents the numerical observations of similar pore structures, as described in 63. It can be seen that the Young modulus obtained for the sample pores are comparable to the simulated values of those modeled based on CT scans of single pore structures in 63 . The variations in the pore geometries can explain the slightly high variations for the plastic collapse stress, or the plateau region of the plots.

$R V E$

In a second set of simulations, entire RVEs with around 25 pores (Figure 32 (a)) are subjected to a uniaxial compression test similar to that studied in [37]. Figure 32(b) illustrates the boundary condition used in the simulation that mimic the setting used in [37. The larger sample size means that no special treatments was considered at the boundaries in the experimental setup, and thus, similar boundary conditions are imposed 


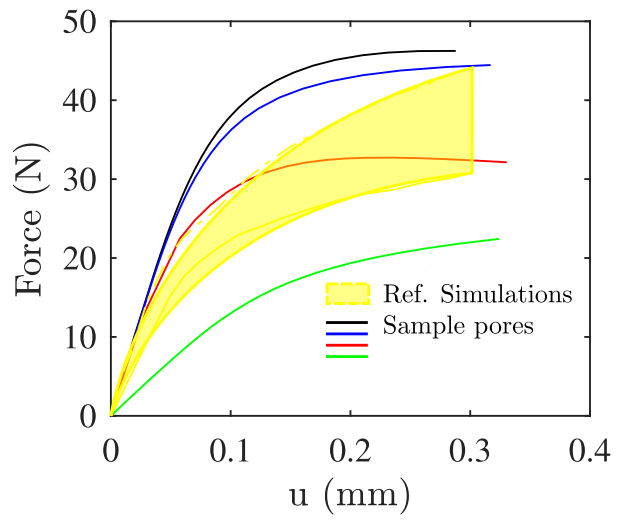

(a)

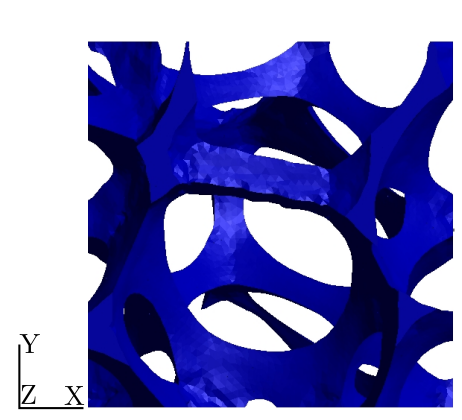

(b)

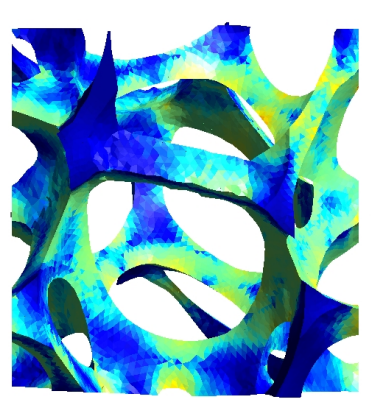

(c)

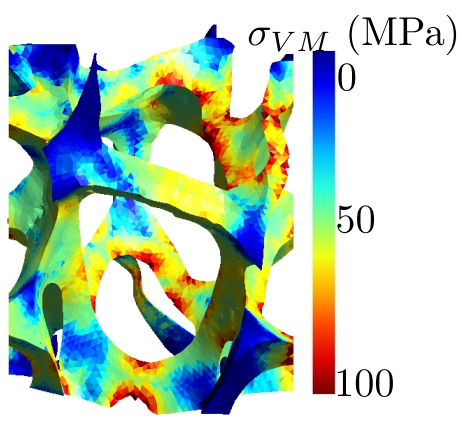

(d)

Figure 31: (a) Comparison of a simulation on a sample single pore generated in the RVE with the average maximum and minimum values in a force displacement plot of a similarly sized pore in 63. (b)-(d) Shows the variation of the von Mises stress [MPa] in the micro-cell with the progress in the simulation of compression test on a single pore. 


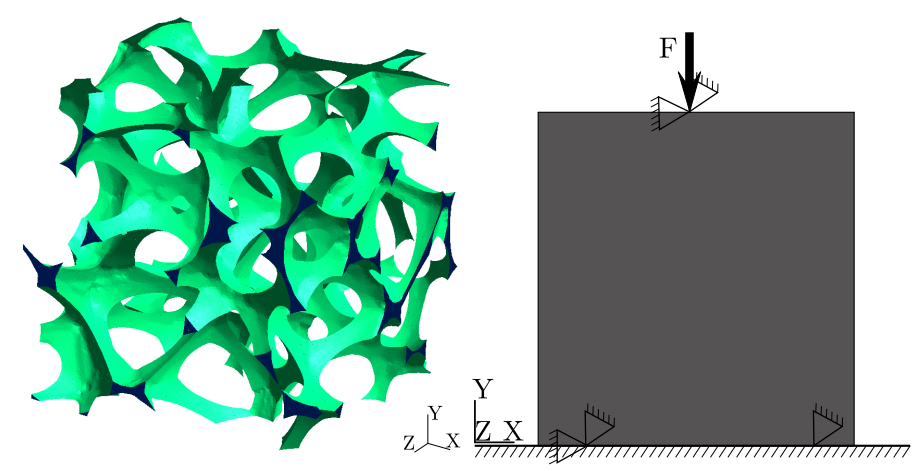

(a)

(b)

Figure 32: (a) An RVE with an initial 100 pores resulting in 25 pores completely inside the RVE boundary after minus-sampling. (b) The boundary conditions imposed on the RVE for the finite element analysis.

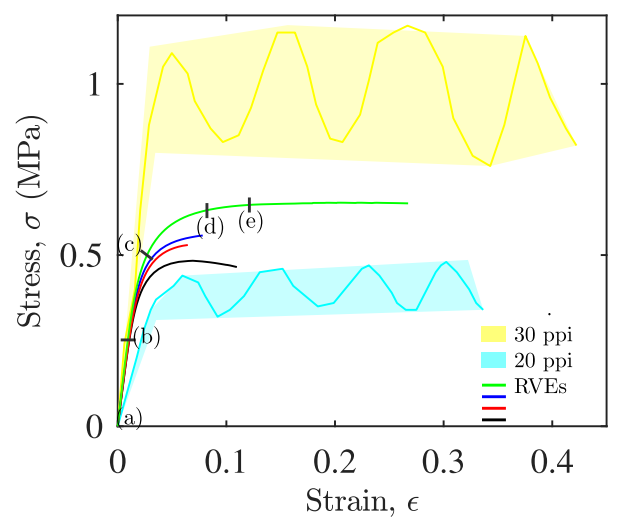

Figure 33: Stress-strain diagram of the simulated RVE in comparison with the values for 20 ppi and 30 ppi taken from [37. Markers (a)-(e) indicate the respective positions in Figure 34 .

on the generated RVE. We, however, note that in order to simulate in an accurate way the experimental test performed on a foam sample having thousands of pores, a multiscale simulation would be required in order to properly represent the boundary conditions and the buckling behavior which occurs layer by layer. The simulation results should thus be seen as illustration of the possibility for the generated foam microstructure to be used in a finite-element framework.

The RVEs here were generated to mimic the properties of a 20 ppi foam. The stressstrain diagram (Figure 33) shows that the curves are very close to the results of the 20 ppi foam taken from 37] and similar properties can be observed for multiple RVEs with very small geometrical variations. It is to be noted that the criterion to enable a simulation with contact conditions was not used here due to the complexity of the geometry. This will need further investigation where the random geometry and the specific point of contact during the plastic failure is established. The deformation of an RVEs is shown in Figure 34 with the von Mises stress plotted on the deformed RVE. 


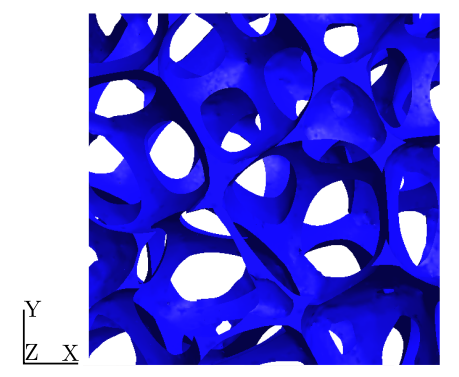

(a)

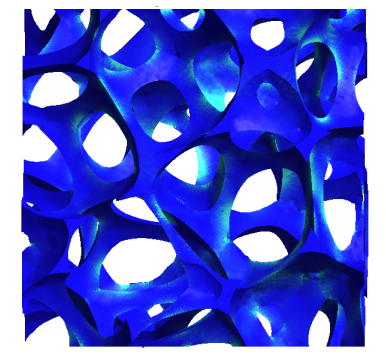

(b)

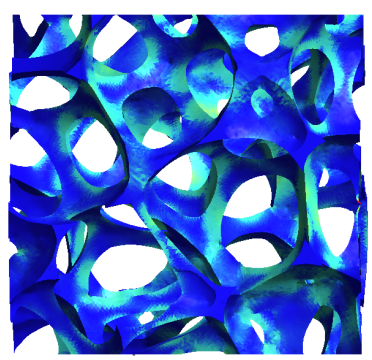

(c)

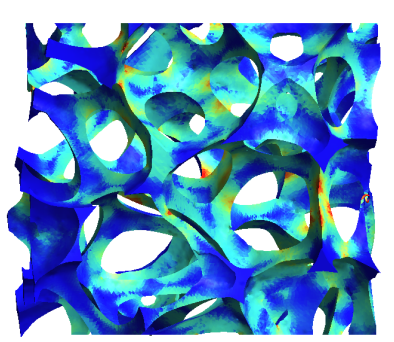

(d)

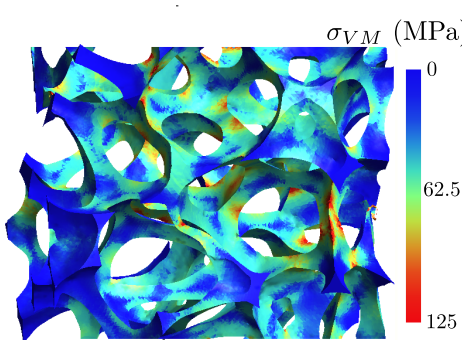

(e)

Figure 34: (a)-(e) displays of the behavior of an RVE with 25 pores under uniaxial compression test. The von Mises stress is plotted on the deformed RVE.

\section{Conclusions}

The present contribution deals with a detailed methodology to produce a representative volume element of a random open-foam metallic structure using distance fields and level sets. An inclusion packing algorithm presented in an earlier work was extended upon to extract RVEs that are morphologically similar in a quantitative way to that of real open-foam samples in terms of the randomness in the geometry of the inclusions, the variation of the strut geometry and the presence of other uncertainties in the extracted model.

Starting from the packing algorithm presented in [40] and operators presented in 41, additional modifications were proposed to generate more subtle shapes of the strut geometry, and to achieve the randomness existing in the real materials, like strut crosssection variation with axis and concavity in the strut cross-section.

A comparison of the extracted geometry with existing Laguerre tessellation-based models was presented to show their geometrical resemblance. A similar comparison with the geometries extracted from CT scans shows that the obtained geometry, in terms of face-to-cell, edge-to-face, strut length distribution, etc, are in close resemblance. The easiness in implementing the variations in the strut geometry using the existing distance fields was also demonstrated, which significantly reduces the extra preprocessing effort needed to obtain the final geometry. A mesh refinement procedure suitable for such geometries was adopted to extract final FE models of the RVEs. A uniaxial compression test of sample RVEs using material parameters established in literature shows the similarities in the mechanical properties of the RVEs to that of open-foam material having 
a similar pore structure.

Future work is necessary to analyze larger foam samples. However since they become computationally more expensive due to the increase in the discretization grid size necessary to capture the randomness of large sized RVEs, this should be conducted within a multiscale framework. It would also be interesting to use the RVEs to define mesoscale phenomenological constitutive models that can account for the local randomness present in the open-foams samples. It might also be noteworthy to compare these with modeling concepts based on surrogate modeling 65] among others.

\section{Acknowledgment}

The authors gratefully acknowledge the financial support from F.R.S-F.N.R.S. under the project number PDR T.0038.16. Computational resources have been provided by the supercomputing facilities of the Consortium des Équipements de Calcul Intensif en Fédération Wallonie Bruxelles (CÉCI) funded by the Fond de la Recherche Scientifique de Belgique (FRS-FNRS).

\section{References}

[1] M. F. Ashby, R. M. Medalist, The mechanical properties of cellular solids, Metallurgical Transactions A 14 (9) (1983) 1755-1769.

[2] L. J. Gibson, M. F. Ashby, Cellular Solids: Structure and Properties, 2nd Edition, Cambridge University Press, Cambridge, 1997. doi:10.1017/CB09781139878326

[3] J. Banhart, Manufacture, characterisation and application of cellular metals and metal foams, Progress in materials science 46 (6) (2001) 559-632.

[4] R. Destefanis, F. Schäfer, M. Lambert, M. Faraud, Selecting enhanced space debris shields for manned spacecraft, International Journal of Impact Engineering 33 (1-12) (2006) 219-230. doi: 10.1016/j.ijimpeng.2006.09.065

[5] G. Ma, Z. Ye, Energy absorption of double-layer foam cladding for blast alleviation, International Journal of Impact Engineering 34 (2) (2007) 329-347. doi:10.1016/j.ijimpeng.2005.07.012.

[6] A. G. Hanssen, L. Enstock, M. Langseth, Close-range blast loading of aluminium foam panels, International Journal of Impact Engineering 27 (6) (2002) 593-618.

[7] P. Di Giorgio, M. Iasiello, A. Viglione, M. Mameli, S. Filippeschi, P. Di Marco, A. Andreozzi, N. Bianco, Numerical Analysis of a Paraffin/Metal Foam Composite for Thermal Storage, Journal of Physics: Conference Series 796 (2017) 012032. doi:10.1088/1742-6596/796/1/012032

[8] W. Li, Z. Qu, Y. He, W. Tao, Experimental and numerical studies on melting phase change heat transfer in open-cell metallic foams filled with paraffin, Applied Thermal Engineering 37 (2012) 1-9. doi:10.1016/j.applthermaleng.2011.11.001

[9] A. Andreozzi, N. Bianco, M. Iasiello, V. Naso, Numerical study of metal foam heat sinks under uniform impinging flow, Journal of Physics: Conference Series 796 (2017) 012002. doi:10.1088/ 1742-6596/796/1/012002

[10] W. H. Shih, F. C. Chou, W. H. Hsieh, Experimental Investigation of the Heat Transfer Characteristics of Aluminum-Foam Heat Sinks With Restricted Flow Outlet, Journal of Heat Transfer 129 (11) (2007) 1554. doi:10.1115/1.2759972

[11] H. Villafán-Vidales, S. Abanades, C. Caliot, H. Romero-Paredes, Heat transfer simulation in a thermochemical solar reactor based on a volumetric porous receiver, Applied Thermal Engineering 31 (16) (2011) 3377-3386. doi:10.1016/j.applthermaleng.2011.06.022

[12] W.-Y. Jang, A. M. Kraynik, S. Kyriakides, On the microstructure of open-cell foams and its effect on elastic properties, International Journal of Solids and Structures 45 (7) (2008) 1845-1875. doi: 10.1016/j.ijsolstr.2007.10.008

[13] J. A. F. Plateau, Statique Expérimentale et Théorique Des Liquides Soumis Aux Seules Forces Moléculaires, Gauthier-Villars, 1873.

[14] A. M. Kraynik, D. A. Reinelt, F. van Swol, Structure of random monodisperse foam, Physical Review E 67 (3). doi:10.1103/PhysRevE.67.031403 
[15] W.-Y. Jang, S. Kyriakides, On the crushing of aluminum open-cell foams: Part I. Experiments, International Journal of Solids and Structures 46 (3-4) (2009) 617-634. doi:10.1016/j.ijsolstr. 2008.09 .008

[16] W.-Y. Jang, S. Kyriakides, On the crushing of aluminum open-cell foams: Part II analysis, International Journal of Solids and Structures 46 (3-4) (2009) 635-650. doi:10.1016/j.ijsolstr. 2008. 10.016

[17] L. Gong, S. Kyriakides, W. Y. Jang, Compressive response of open-cell foams. Part I: Morphology and elastic properties, International Journal of Solids and Structures 42 (5) (2005) 1355-1379. doi:10.1016/j.ijsolstr.2004.07.023

[18] A. M. Hodge, D. C. Dunand, Measurement and modeling of creep in open-cell NiAl foams, Metallurgical and Materials Transactions A 34 (10) (2003) 2353-2363.

[19] A. Jung, H. Natter, S. Diebels, E. Lach, R. Hempelmann, Nanonickel Coated Aluminum Foam for Enhanced Impact Energy Absorption, Advanced Engineering Materials 13 (1-2) (2011) 23-28. doi:10.1002/adem.201000190

[20] C. Veyhl, I. Belova, G. Murch, T. Fiedler, Finite element analysis of the mechanical properties of cellular aluminium based on micro-computed tomography, Materials Science and Engineering: A 528 (13-14) (2011) 4550-4555. doi:10.1016/j.msea.2011.02.031

[21] T. Fiedler, I. Belova, G. Murch, $\mu$-CT-based finite element analysis on imperfections in open-celled metal foam: Mechanical properties, Scripta Materialia 67 (5) (2012) 455-458. doi:10.1016/j. scriptamat.2012.06.002

[22] T. Fiedler, E. Solórzano, F. Garcia-Moreno, A. Öchsner, I. Belova, G. Murch, Computed tomography based finite element analysis of the thermal properties of cellular aluminium, Materialwissenschaft und Werkstofftechnik 40 (3) (2009) 139-143. doi:10.1002/mawe.200900419

[23] M. D. Montminy, A. R. Tannenbaum, C. W. Macosko, The 3D structure of real polymer foams Journal of Colloid and Interface Science 280 (1) (2004) 202-211. doi:10.1016/j.jcis.2004.07.032

[24] W. Thomson, LXIII. On the division of space with minimum partitional area, The London, Edinburgh, and Dublin Philosophical Magazine and Journal of Science 24 (151) (1887) 503-514.

[25] D. Weaire, R. Phelan, A counter-example to Kelvin's conjecture on minimal surfaces, Philosophical Magazine Letters 69 (2) (1994) 107-110. doi:10.1080/09500839408241577

[26] F. Wan, M.-P. Tran, C. Leblanc, E. Béchet, E. Plougonven, A. Léonard, C. Detrembleur, L. Noels, J.-M. Thomassin, V.-D. Nguyen, Experimental and computational micro-mechanical investigations of compressive properties of polypropylene/multi-walled carbon nanotubes nanocomposite foams, Mechanics of Materials 91 (2015) 95-118. doi:10.1016/j.mechmat.2015.07.004

[27] J. L. Grenestedt, K. Tanaka, Influence of cell shape variations on elastic stiffness of closed cell cellular solids, Scripta Materialia 40 (1) (1998) 71-77. doi:10.1016/S1359-6462(98)00401-1

[28] M. W. D. Van Der Burg, V. Shulmeister, E. Van Der Geissen, R. Marissen, On the Linear Elastic Properties of Regular and Random Open-Cell Foam Models, Journal of Cellular Plastics 33 (1) (1997) 31-54. doi:10.1177/0021955X9703300103

[29] Z. Fan, Y. Wu, X. Zhao, Y. Lu, Simulation of polycrystalline structure with Voronoi diagram in Laguerre geometry based on random closed packing of spheres, Computational Materials Science 29 (3) (2004) 301-308. doi:10.1016/j.commatsci.2003.10.006

[30] C. Lautensack, Fitting three-dimensional Laguerre tessellations to foam structures, Journal of Applied Statistics 35 (9) (2008) 985-995. doi:10.1080/02664760802188112

[31] C. Redenbach, Microstructure models for cellular materials, Computational Materials Science 44 (4) (2009) 1397-1407. doi:10.1016/j.commatsci.2008.09.018

[32] I. Vecchio, C. Redenbach, K. Schladitz, Angles in Laguerre tessellation models for solid foams, Computational Materials Science 83 (2014) 171-184. doi:10.1016/j.commatsci.2013.11.017.

[33] A. Bezrukov, M. Bargiel, D. Stoyan, Statistical Analysis of Simulated Random Packings of Spheres, Part. Part. Syst. Charact. 19 (2) (2002) 111-118. doi:10.1002/1521-4117(200205) 19:2<111: : AID-PPSC111>3.0.CO;2-M

[34] A. Kraynik, D. Reinelt, F. van Swol, Structure of Random Foam, Physical Review Letters 93 (20). doi:10.1103/PhysRevLett.93.208301

[35] A. M. Kraynik, The Structure of Random Foam, Adv. Eng. Mater. 8 (9) (2006) 900-906. doi: 10.1002/adem.200600167

[36] I. Vecchio, C. Redenbach, K. Schladitz, A. M. Kraynik, Improved models of solid foams based on soap froth, Computational Materials Science 120 (2016) 60-69. doi:10.1016/j.commatsci.2016. 03.029

[37] A. Jung, S. Diebels, Microstructural characterisation and experimental determination of a multiaxial yield surface for open-cell aluminium foams, Materials \& Design 131 (2017) 252-264. doi:10.1016/ 
j.matdes.2017.06.017

[38] L. Gong, S. Kyriakides, Compressive response of open cell foams Part II: Initiation and evolution of crushing, International Journal of Solids and Structures 42 (5-6) (2005) 1381-1399. doi:10.1016/ j.ijsolstr.2004.07.024

[39] W.-Y. Jang, S. Kyriakides, A. M. Kraynik, On the compressive strength of open-cell metal foams with Kelvin and random cell structures, International Journal of Solids and Structures 47 (21) (2010) 2872-2883. doi:10.1016/j.ijsolstr.2010.06.014.

[40] B. Sonon, B. François, T. J. Massart, A unified level set based methodology for fast generation of complex microstructural multi-phase RVEs, Computer Methods in Applied Mechanics and Engineering 223-224 (2012) 103-122. doi:10.1016/j.cma.2012.02.018.

[41] B. Sonon, B. François, T. J. Massart, An advanced approach for the generation of complex cellular material representative volume elements using distance fields and level sets, Computational Mechanics 56 (2) (2015) 221-242. doi:10.1007/s00466-015-1168-8.

[42] P.-O. Persson, G. Strang, A simple mesh generator in MATLAB, SIAM review 46 (2) (2004) 329 345 .

[43] C. Perrot, R. Panneton, X. Olny, Periodic unit cell reconstruction of porous media: Application to open-cell aluminum foams, Journal of Applied Physics 101 (11) (2007) 113538. doi:10.1063/1. 2745095 .

[44] J. C. Kim, D. M. Martin, C. S. Lim, Effect of rearrangement on simulated particle packing, Powder technology 126 (3) (2002) 211-216.

[45] K. Bagi, An algorithm to generate random dense arrangements for discrete element simulations of granular assemblies, Granular Matter 7 (1) (2005) 31-43. doi:10.1007/s10035-004-0187-5

[46] S. J. Hollister, N. Kikuchi, A comparison of homogenization and standard mechanics analyses for periodic porous composites, Computational Mechanics 10 (2) (1992) 73-95.

[47] A. J. Baddeley, Spatial sampling and censoring, Stochastic geometry: likelihood and computation 2 (1999) 37-78.

[48] B. Sonon, On advanced techniques for generation and discretization of the microstructure of complex heterogeneous materials, Ph.D. thesis, Université libre de Bruxelles (2014).

[49] V.-D. Nguyen, L. Noels, Computational homogenization of cellular materials, International Journal of Solids and Structures 51 (11-12) (2014) 2183-2203. doi:10.1016/j.ijsolstr.2014.02.029

[50] E. Andrews, W. Sanders, L. J. Gibson, Compressive and tensile behaviour of aluminum foams, Materials Science and Engineering: A 270 (2) (1999) 113-124. doi:10.1016/S0921-5093(99)00170-7

[51] K. Ehab Moustafa Kamel, B. Sonon, T. J. Massart, An integrated approach for the generation and conformal discretization of complex inclusion-based microstructures, In Pressdoi:10.1007/ s00466-019-01693-4

[52] B. Wintiba, B. Sonon, K. Ehab Moustafa Kamel, T. J. Massart, An automated procedure for the generation and conformal discretization of 3D woven composites RVEs, Composite Structures 180 (2017) 955-971. doi:10.1016/j.compstruct.2017.08.010

[53] T. Möller, A Fast Triangle-Triangle Intersection Test, Journal of Graphics Tools 2 (1997) 25-30. doi:10.1080/10867651.1997.10487472

[54] G. Bernstein, D. Fussell, Fast, exact, linear booleans, in: Computer Graphics Forum, Vol. 28, Wiley Online Library, 2009, pp. 1269-1278.

[55] W. E. Lorensen, H. E. Cline, Marching cubes: A high resolution 3D surface construction algorithm, ACM Press, 1987, pp. 163-169. doi:10.1145/37401.37422

[56] J. R. Shewchuk, Constrained Delaunay Tetrahedralizations and Provably Good Boundary Recovery, IMR, 2002, p. 12 .

[57] H. Si, TetGen, a Delaunay-Based Quality Tetrahedral Mesh Generator, ACM Transactions on Mathematical Software 41 (2) (2015) 1-36. doi:10.1145/2629697

[58] C. B. Barber, D. P. Dobkin, H. Huhdanpaa, The quickhull algorithm for convex hulls, ACM Transactions on Mathematical Software 22 (4) (1996) 469-483. doi:10.1145/235815.235821

[59] C. Monnereau, B. Prunet-Foch, M. Vignes-Adler, Topology of slightly polydisperse real foams, Physical Review E 63 (6). doi:10.1103/PhysRevE.63.061402.

[60] S. Kanaun, O. Tkachenko, Mechanical Properties of Open Cell Foams: Simulations by Laguerre Tesselation Procedure, International Journal of Fracture 140 (1-4) (2006) 305-312. doi:10.1007/ s10704-006-0112-5

[61] K. A. Brakke, Minimal surfaces, corners, and wires, Journal of Geometric Analysis 2 (1) (1992) $11-36$.

[62] A. Liebscher, Laguerre approximation of random foams, Philosophical Magazine 95 (25) (2015) 2777-2792. doi:10.1080/14786435.2015.1078511 
[63] S. Heinze, T. Bleistein, A. Düster, S. Diebels, A. Jung, Experimental and numerical investigation of single pores for identification of effective metal foams properties: Experimental and numerical investigation of single pores for identification of effective metal foams properties, ZAMM - Journal of Applied Mathematics and Mechanics / Zeitschrift für Angewandte Mathematik und Mechanik 98 (5) (2018) 682-695. doi:10.1002/zamm. 201700045

[64] V.-D. Nguyen, L. Wu, L. Noels, Unified treatment of microscopic boundary conditions and efficient algorithms for estimating tangent operators of the homogenized behavior in the computational homogenization method, Computational Mechanics 59 (3) (2017) 483-505. doi:10.1007/ s00466-016-1358-z

[65] M. Marvi-Mashhadi, C. S. Lopes, J. LLorca, Surrogate models of the influence of the microstructure on the mechanical properties of closed- and open-cell foams, Journal of Materials Science 53 (18) (2018) 12937-12948. doi:10.1007/s10853-018-2598-4 